\title{
CONTROL COMMUNICATION COMPLEXITY OF NONLINEAR SYSTEMS*
}

\author{
WING SHING WONG $^{\dagger}$ AND JOHN BAILLIEUL ${ }^{\ddagger}$
}

\begin{abstract}
The interaction of information and control has been a topic of interest to system theorists that can be traced back at least to the 1950's when the fields of communications, control, and information theory were new but developing rapidly. Recent advances in our understanding of this interplay have emerged from work on the dynamical effect of state quantization together with results connecting communication channel data rates and system stability. Although this work has generated considerable interest, it has been centrally concerned with the relationship between control system performance and feedback information processing rates while ignoring the complexity (i.e. the cost of information processing). The concepts of communication and computation complexity of a controlled dynamical system based on digitized information lie in what is largely an uncharted area. In our recent work an attempt was made to explore this area by introducing a new measure of communication complexity for a two-player distributed control system. This complexity is named control communication complexity (CCC). It is based on the communication complexity concept defined in distributed computing and seeks to connect the complexity of information exchange over finite bandwidth channels with the control system dynamics. The purpose of the present paper is to extend the study of control communication complexity to an interesting class of continuous-time control systems that have appeared in the recent literature dealing with quantum communication and control systems. An interesting aspect of this extension is that it brings together heretofore independent research themes that have been prominent in the research career of Roger Brockett.
\end{abstract}

1. Introduction. In his seminal paper, [Yao1] defined the concept of communication complexity with the aim of understanding certain cost aspects of information exchange between processors that cooperate to perform computations. For an introduction to this theory one can refer to the monograph Communication Complexity by Kushilevitz and Nisan ([KuNi]). In recent work [Wong], one of the authors investigated similar ideas in the context of digital finite communication bandwidth control, where one studies control system dynamics wherein multiple agents collaboratively provide inputs to a control system in order to achieve a common objective that no single agent could achieve alone. The information that is exchanged by agents that cooperatively steer a control system to a given objective determines what we shall call the control communication complexity of the problem.

The purpose of the present paper is to explore this circle of ideas in the context of some well-known work of Roger Brockett - especially work he has done over sev-

*Dedicated to Roger Brockett on the occasion of his 70th birthday. Research work is partially supported by a Grant from the Research Grants Council of the Hong Kong Special Administrative Region under the project 417207. Also, JB acknowledges support from ODDR\& E MURI07 Program Grant Number FA9550-07-1-0528, and the National Science Foundation ITR Program Grant Number DMI-0330171.

${ }^{\dagger}$ CUHK. E-mail: wswong@ie.cuhk.edu.hk

${ }^{\ddagger}$ B.U. E-mail: johnb@bu.edu 
eral decades on geometric optimal control. Over the course of his career, Brockett made seminal contributions to modern control theory, the most important of which was probably his founding the field of geometric nonlinear control. It is striking that while he has remained a leader in the field, he has also introduced important new perspectives in other parts of the discipline - one notable example being in what might be called information-based control. This paper poses a problem that is directly related to both nonlinear optimal control and the information-exchange in the distributed control of nonlinear systems. In general terms, we consider control systems of the form

$$
\left\{\begin{array}{l}
\dot{x}=f(x, u) \\
y=h(x) .
\end{array}\right.
$$

evolving on some state manifold $M$ of dimension $n$ and in which the dimension $m$ of the input $u=\left(u_{1}, \ldots, u_{m}\right)$ is greater than one. The present paper focuses to a large extent on a distributed version of the problem in which entries $u_{i}(\cdot)$ 's in the control vector are determined by independent agents who collaborate to achieve specified control objectives. (One could also consider a related problem in which adversarial agents "anti-collaborate" in an effort to foil each other from achieving control objectives. This problem will not be considered here.) The objectives, finite in number, are expressed as terminal states $h\left(x_{1}(T)\right), \ldots, h\left(x_{N}(T)\right)$, and we assume that $N$ can be expressed as the product of $m$ positive integers $k_{1} k_{2} \cdots k_{m}$. The optimal control problem we wish to consider seeks to find $N$ choices of control inputs $\left(u_{1, i_{1}}, \ldots, u_{m, i_{m}}\right)$ where $1 \leq i_{j} \leq k_{j}, j=1, \ldots, m$, with each $m$-tuple $\xi=\left(i_{1}, \ldots, i_{m}\right)$ identifing a unique control objective $h\left(x_{\xi}(T)\right)$ from our list. The controls are designed so that each $m$-tuple $\left(u_{1, i_{1}}, \ldots, u_{m, i_{m}}\right)$ steers (1.1) from a common initial state $x_{0}$ to the goal state $x_{\xi}(T)$ in such a way that

$$
\eta=\int_{0}^{T} \sum_{j=1}^{k_{1}} u_{1, j}(t)^{2}+\cdots+\sum_{j=1}^{k_{m}} u_{m, j}(t)^{2} d t
$$

is minimized. Note that in general the sum of the number of possible control choices over the components of the inputs, $k_{1}+\cdots+k_{m}$ is less than the number $N=k_{1} \cdots k_{m}$ of goal states. It is also the case that the problem remains interesting if not all goal states are distinct from one another - in which case some combinations of the control inputs will lead to the same terminal state. At this level of generality, the problem has some of the flavor of Brockett's very recent work on design and optimization techniques for families of control system trajectories. We refer, for instance, to [RWB4] and [RWB5]. It is in the first of these references that the term "standard parts" is used in describing an approach to control system design. We shall also adopt this terminology and refer to the above as the standard parts optimization problem.

The present paper is concerned with a restricted version of the problem in which $m=2$. We consider the relaxed objective of choosing a set of control inputs that 
will drive an output function $h(\cdot)$ to a set of goal values $\mathfrak{h}_{i j}$ prescribed by an $n_{1} \times n_{2}$ matrix $\mathbf{H}$. We are thus concerned with the problem of finding a choice of $n_{1}$ scalar inputs $u_{i}$ and $n_{2}$ inputs $v_{j}$ such that together $u_{i}$ and $v_{j}$ steer

$$
\dot{x}(t)=a(x(t), u(t), v(t)), \quad y(t)=h(x(t))
$$

from $x(0)=x_{0} \in M$ to $x(T)$ such that $h(x(T))=\mathfrak{h}_{i j}$ and such that the collective cost

$$
\int_{0}^{T} \sum_{i=1}^{n_{1}} u_{i}(t)^{2}+\sum_{j=1}^{n_{2}} v_{j}(t)^{2} d t
$$

is minimized. The problem can be viewed in different ways. It can be seen as an extension of the optimal control problems on manifolds that were of interest to Roger Brockett several decades ago. (See specifically, the 1973 paper "Lie Theory and Control Systems Defined on Spheres," [RWB0].) Our own goal in the research discussed below is to understand how the performance index (1.3) measures the cost of performing a computation and also the complexity of the communication taking place between agents in executing a prescribed computation. While physicists have been concerned for some time with the minimum energy needed to perform computations (See e.g. [Feynman].), there has been very little written about how these problems might be formulated in terms of optimal control. The goal of our study of control communication complexity $(\mathrm{CCC})$ is to understand the collaboration dynamics and information exchange that enable a system with distributed inputs to meet given objectives while minimizing some collective measure of performance. For the case of $2 \times 2$ goal matrices $\mathbf{H}$, we solve a parametric version of (1.2)-(1.3) in the case that (1.2) is the so-called Heisenberg system and the control inputs are sinusoids parameterized by phase and amplitude. Sections 4,5 , and 6 describe the main results. In a related paper ([WongBa1]), we treat the case of $n_{1} \times n_{2}$ goal matrices and control inputs that are represented by Fourier series.

In Yao's original paper [Yao1] and in much of the work that has followed, the focus had been on the number of bits of information that must be exchanged between collaborators (Alice and Bob) in order to efficiently compute the value of a Boolean function $f(x, y)$ assuming Alice initially knows only the value of $x$, and Bob initially knows only the value of $y$. Efficiency is measured in terms of the number of bits of information that Alice and Bob exchange regarding the values of their respective variables $x$ and $y$. In [Wong], the focus of the extension to control communication complexity remains on collaborative tasks being completed through the sharing between agents of finite numbers of bits of information. In the present paper, we pose problems of multiagent collaborative control of systems prescribed by nonlinear differential equations. A question that comes immediately to mind is how to model information exchange among agents in this setting. As we shall show in the remainder of the paper, there is an interesting class of nonlinear control systems that have 
been extensively studied and written about by Brockett, for which the system objectives are achieved using simple closed curves as control inputs. In order to make this precise, we shall focus most of the paper on two and three-dimensional specializations of (1.1) in which there are two independent control inputs. In the next section, we introduce a number of two-input nonlinear control systems. It is noted that these can arise as models in both classical and quantum mechanics (spin systems). Section 3 introduces a general class of distributed control systems and defines the concept of control communication complexity. In Section 4, the distributed control control is specialized to a particular two-input system that is introduced in Section 2. The control complexity of this model is studied in Section 5. Section 6 relates the control communication complexity problem to the standard parts optimization problem. For the model treated in Sections 4 and 5, a parametric solution to the standard parts optimization problem is given in terms of what we call phased-loop inputs. Section 7 addresses the challenges of control communication in the case that the loop inputs are subject to phase uncertainty.

\section{Some Optimal Control Problems on 2 and 3 Dimensional State Man-}

ifolds. Consider a nonlinear system with two independent controls whose evolution is described by the equation

$$
\dot{x}=u_{1} f_{1}(x)+u_{2} f_{2}(x) ; x(0)=x_{0}
$$

where $x$ is a coordinate representation of a point on a three-dimensional manifold $M$, and for all $x$ in an open neighborhood of $x_{0} \in M$, the three-elements set $\left\{f_{1}(x), f_{2}(x)\right.$, $\left.\left[f_{1}(x), f_{2}(x)\right]\right\}$ is a basis for the tangent space $T_{x} M$. Here $\left[f_{1}(x), f_{2}(x)\right]$ is the standard Lie bracket of vectorfields:

$$
\left[f_{1}, f_{2}\right]=\frac{\partial f_{1}}{\partial x} f_{2}-\frac{\partial f_{2}}{\partial x} f_{1} .
$$

Specific examples of manifolds and systems of interest include the following.

EXAMPLE 2.1. $M=S U(2)$, the group of 2 by 2 unitary matrices. The set of two by two matrices of the form

$$
\left(\begin{array}{cc}
x+i y & -z+i w \\
z+i w & x-i y
\end{array}\right)
$$

where $x^{2}+y^{2}+w^{2}+z^{2}=1$ is the standard representation of the special unitary group $S U(2)$. It is a three dimensional manifold whose topology is the same as that of the 3 -sphere in $\mathbb{R}^{4}$.

We note that the tangent spaces at all points $X \in S U(2)$ are isomorphic to the tangent space at the identity, which we denote as usual by $s u(2)$. Thus, vectorfields on $S U(2)$ may be represented in terms of elements in $s u(2)$. Letting $A \in s u(2)$, the corresponding right-invariant vectorfield at $X \in M(=S U(2))$ is $A X$. For details on 
the connection between the Lie algebra $s u(2)$ and the Lie algebra of vectorfields on $S U(2)$, we refer to standard texts, such as [Var]. One choice of basis for $s u(2)$ is

$$
A_{1}=\left(\begin{array}{cc}
0 & i / 2 \\
i / 2 & 0
\end{array}\right), A_{2}=\left(\begin{array}{cc}
0 & -1 / 2 \\
1 / 2 & 0
\end{array}\right), A_{3}=\left(\begin{array}{cc}
i / 2 & 0 \\
0 & -i / 2
\end{array}\right)
$$

in terms of which a control system of the form (2.1) is given by

$$
\dot{X}=u_{1} A_{1} X+u_{2} A_{2} X, X(0)=I .
$$

The Lie bracket operation(2.2) when specialized to $M=S U(2)$ is then represented by the matrix Lie bracket $[A, B]=A B-B A$. For the chosen basis, it is easy to verify that

$$
\left[A_{1}, A_{2}\right]=A_{3} .
$$

The chosen basis for su(2) may be expressed in terms of the so-called Pauli spin matrices: $\sigma_{j}=i A_{j}(j=1,2,3$, and $i=\sqrt{-1})$. Any element in $X \in S U(2)$ can be written in the form

$$
X=e^{i \sigma_{1} \theta_{1}} e^{i \sigma_{2} \theta_{2}} e^{i \sigma_{3} \theta_{3}}
$$

where $0 \leq \theta_{j}<2 \pi$ for $j=1,3$, and $0 \leq \theta_{2} \leq \pi$. As noted in [Holevo] and elsewhere, $S U(2)$ plays an important role in modeling quantum bits (qubits), and in such applications, elements of the group are typically represented in terms of the Pauli spin matrices.

EXAMPLE 2.2. $M=S O(3)$, the special orthogonal group of rotations of the 2-sphere. Again the tangent space at any point in this group manifold is isomorphic as a Lie algebra to the tangent space at the identity, which we label in the usual way as $s o(3)$. The algebraic structure of $s o(3)$ is easily discovered. Let $A \in s o(3)$ and let $R(t) \in S O(3)$ be a curve such that $R(0)=I$ and $\dot{R}(0)=A$. By differentiating both sides of the equation $R(t) R(t)^{T} \equiv I$ and evaluating these at 0 , it is easily seen that $A$ must be skew-symmetric - verifying the well-known fact that so(3) is the matrix Lie algebra of $3 \times 3$ skew-symmetric matrices. It will follow easily from the next proposition that every $3 \times 3$ skew-symmetric matrix is the tangent at the identity to some curve in $S O(3)$, and hence $s o(3)$ contains the set

$$
\left\{\left(\begin{array}{ccc}
0 & -\gamma & \beta \\
\gamma & 0 & -\alpha \\
-\beta & \alpha & 0
\end{array}\right): \alpha, \beta, \gamma \in \mathbb{R}\right\}
$$

The following shows the relationship between the above set and elements of $S O(3)$ via the well-known Rodrigues' formula. 
Proposition 2.1. (Rodrigues' formula) [RWB1] Let

$$
\Omega=\left(\begin{array}{ccc}
0 & -\gamma & \beta \\
\gamma & 0 & -\alpha \\
-\beta & \alpha & 0
\end{array}\right)
$$

be an arbitrary skew-symmetric matrix. Then we have the following closed-form expression for the matrix exponential

$$
e^{\Omega}=I+\Omega^{\prime} \sin \lambda+\Omega^{\prime 2}(1-\cos \lambda)
$$

where $\lambda=\sqrt{\alpha^{2}+\beta^{2}+\gamma^{2}}$ and $\Omega^{\prime}=\Omega / \lambda$.

The geometry of $S O(3)$. There is an elegant way to visualize $S O(3)$ based on Rodrigues' formula (2.4) and the observation that the exponential mapping is onto from $s o(3)$ to $S O(3)$. The later result is known in various forms (see for example [TaKr].) To facilitate subsequent discussions, we present the following version with a proof.

Proposition 2.2. Let e $: s o(3) \rightarrow S O(3)$ be given by the formula (2.4). For all $X \in S O(3)$,

1. $-1 \leq \operatorname{tr}(X) \leq 3$;

2. if $-1<\operatorname{tr}(X)<3$, there is a unique angle $\theta, 0<\theta<\pi$, and a unique skew symmetric matrix

$$
\Omega=\left(\begin{array}{ccc}
0 & -\gamma & \beta \\
\gamma & 0 & -\alpha \\
-\beta & \alpha & 0
\end{array}\right)
$$

with $\alpha^{2}+\beta^{2}+\gamma^{2}=1$ such that

$$
e^{\Omega \theta}=X, \text { and }
$$

3. if $\operatorname{tr}(X)=3$, then $X=I$ (the $3 \times 3$ identity matrix). If $\operatorname{tr}(X)=-1$, then we may solve (2.5) for $\theta=\pi$. The unit vector $(\alpha, \beta, \gamma)$ is also determined by (2.5), but only up to multiplication by \pm 1 .

Proof. (1) Define the two "Euler" matrices

$$
\mathrm{R}_{z}(\alpha)=\left(\begin{array}{ccc}
\cos \alpha & -\sin \alpha & 0 \\
\sin \alpha & \cos \alpha & 0 \\
0 & 0 & 1
\end{array}\right) \quad \mathrm{R}_{y}(\alpha)=\left(\begin{array}{ccc}
\cos \alpha & 0 & \sin \alpha \\
0 & 1 & 0 \\
-\sin \alpha & 0 & \cos \alpha
\end{array}\right) .
$$

It is well-known that any element $X \in S O(3)$ can be written as the product $X=$ $\mathrm{R}_{z}(\phi) \mathrm{R}_{y}(\xi) \mathrm{R}_{z}(\psi)$ where $0 \leq \phi, \psi<2 \pi$ and $0 \leq \xi \leq \pi$. Using this representation of $X$, we have $x_{11}+x_{22}+x_{33}=\cos \xi+\cos \xi \cos (\phi+\psi)+\cos (\phi+\psi)$. Now if $\operatorname{tr}(X)<0$, 
at least one of $\cos \xi$ and $\cos (\phi+\psi)$ must also be less than 0 . Assume, without loss of generality, that $\cos \xi<0$. Writing $\operatorname{tr}(X)=a+(1+a) b$ where $a=\cos \xi$ and $b=\cos (\phi+\psi)$, we see immediately that because $1+a \geq 0$ this is always larger than or equal to -1 . The inequality $\operatorname{tr}(X) \leq 3$ is an immediate consequence of this Euler-angle representation. This proves (1)

To prove (2), note that from Proposition 1.1, it follows that the expansion of the left hand side of (2.5) yields

$$
e^{\Omega \theta}=I+\sin \theta \Omega+(1-\cos \theta) \Omega^{2} .
$$

To solve the matrix equation (2.5), we sum the diagonal entries on both sides

$$
x_{11}+x_{22}+x_{33}=1+2 \cos \theta .
$$

The cosine function defines a one-to-one mapping of the interval $[0, \pi]$ onto $[-1,1]$, and hence there is a unique value of $\theta \in[0, \pi]$ given by

$$
\theta=\arccos \left[\frac{x_{11}+x_{22}+x_{33}-1}{2}\right] .
$$

Note that if $\theta=0, \pi$, the problem of finding $\alpha, \beta$, and $\gamma$ is indeterminate. For $\theta \in(0, \pi)$, however, we may solve explicitly

$$
\alpha=\frac{x_{32}-x_{23}}{2 \sin \theta}, \beta=\frac{x_{13}-x_{31}}{2 \sin \theta}, \gamma=\frac{x_{21}-x_{12}}{2 \sin \theta} .
$$

To prove (3), note that if $\theta=0, X$ must be the identity matrix. If, on the other hand, $\theta=\pi, X$ must be a symmetric matrix, and the equations

$$
2 \alpha \beta=x_{12}=x_{21} \quad 2 \alpha \gamma=x_{13}=x_{31} \quad 2 \beta \gamma=x_{23}=x_{32}
$$

can be solved for the entries in a unit vector $(\alpha, \beta, \gamma)$ which is unique only up to multiplication by \pm 1 . This explicit solution proves the proposition.

The geometric content of the proposition is that every element in $S O(3)$ can be visualized as a rotation by some angle $\theta$ about a unit vector $(\alpha, \beta, \gamma)$. These quantities can be viewed as points in a solid ball of radius $\pi$ in $\mathbb{R}^{3}$. In this representation, an element corresponding to the parameters $(\alpha, \beta, \gamma)$, and $\theta$ will be associated to the point in the radius- $\pi$ ball lying $\theta$-units from the origin in the direction of the unit vector $(\alpha, \beta, \gamma)$. Note, that in light of Proposition 1.2(3), the antipodal points on the surface of the radius- $\pi$ ball correspond to the same element of $S O(3)$. Since these points cannot be distinguished from one another, we identify them. Through this construction, we see that $S O(3)$ is diffeomorphic to the three dimensional real projective plane $\mathbb{R} P^{3}$.

The relationship of $S O(3)$ to $S U(2)$. There is a group homomorphism from $S U(2)$ and $S O(3)$, and a Lie algebra isomorphism between $s u(2)$ and $s o(3)$ (see for example 
[CaMa].) This isomorphism is given explicitly by

$$
\left(\begin{array}{ccc}
0 & -\gamma & \beta \\
\gamma & 0 & -\alpha \\
-\beta & \alpha & 0
\end{array}\right) \leftrightarrow \frac{1}{2}\left(\begin{array}{cc}
i \gamma & -\beta+i \alpha \\
\beta+i \alpha & -i \gamma
\end{array}\right)
$$

Choosing the canonical so(3) basis

$$
B_{1}=\left(\begin{array}{ccc}
0 & 0 & 0 \\
0 & 0 & -1 \\
0 & 1 & 0
\end{array}\right), B_{2}=\left(\begin{array}{ccc}
0 & 0 & 1 \\
0 & 0 & 0 \\
-1 & 0 & 0
\end{array}\right), B_{3}=\left(\begin{array}{ccc}
0 & -1 & 0 \\
1 & 0 & 0 \\
0 & 0 & 0
\end{array}\right),
$$

this isomorphism is specified in terms of the bases by $B_{j} \leftrightarrow A_{j}(j=1,2,3)$. From either the isomorphism or a direct calculation we find that

$$
B_{3}=\left[B_{1}, B_{2}\right] .
$$

While there is a natural one-to-one correspondence between the Lie algebras of so(3) and $s u(2)$, the relationship between the Lie groups $S O(3)$ and $S U(2)$ is less straightforward. Indeed, while $S U(2)$ is diffeomorphic to the 3 -sphere, we have seen above that $S O(3)$ is diffeomorphic to the 3 -dimensional projective plane. Thus, while $S U(2)$ is simply connected, $S O(3)$ is not. $S U(2)$ is a double covering of $S O(3)$, as may be illustrated by the Rodrigues' formula prescribing the (surjective) exponential mapping $s u(2) \rightarrow S U(2)$ :

$$
\begin{aligned}
& \exp \left[\frac{1}{2}\left(\begin{array}{cc}
i \gamma & -\beta+i \alpha \\
\beta+i \alpha & -i \gamma
\end{array}\right) \theta\right]= \\
& \left(\begin{array}{cc}
\cos (\theta / 2) & 0 \\
0 & \cos (\theta / 2)
\end{array}\right)+\left(\begin{array}{cc}
i \gamma & -\beta+i \alpha \\
\beta+i \alpha & -i \gamma
\end{array}\right) \sin (\theta / 2) .
\end{aligned}
$$

As $\theta$ is varied from 0 to $2 \pi$ in (2.5), a noncontractable loop beginning and ending at the identity of $S O(3)$ is traced. As $\theta$ is varied from 0 to $2 \pi$ in (2.6), however, a non-closed curve from $I$ to $-I$ is traced in $S U(2)$. By continuing $\theta$ from $2 \pi$ to $4 \pi$, the corresponding curve in $S U(2)$ returns to $I$, while the curve in $S O(3)$ is traced a second time. In terms of this double covering, a second construction of $S O(3)$ can be obtained by identifying all pairs of elements $X$ and $-X$ in $S U(2)$. Since $S U(2)$ is diffeomorphic to the 3 -sphere, $S^{3} \subset \mathbb{R}^{4}$, this is an alternative way to view $S O(3)$ as real projective 3 -space, $\mathbb{R} P^{3}$.

In terms of these matrices, the two-input control system corresponding to (2.3) is given by

$$
\dot{X}=u_{1} B_{1} X+u_{2} B_{2} X, X(0)=I .
$$

For this system, we pose the following optimal control problem: Find controls $u_{1}(\cdot)$, $u_{2}(\cdot)$ that steer $(2.7)$ from $I$ to $X \in S O(3)$ in $T>0$ units of time such that the 
integral

$$
\eta=\int_{0}^{T} u_{1}(t)^{2}+u_{2}(t)^{2} d t
$$

is minimized.

TheOREM 2.1. (Baillieul, 1978) Given the preceding optimal control problem let $u_{1}^{0}(t), u_{2}^{0}(t)$ be optimal control inputs to (2.7). There exists a differentiable function $\omega(t)$ such that the following differential equation is satisfied

$$
\left(\begin{array}{ccc}
0 & -\dot{\omega} & \dot{u}_{2} \\
\dot{\omega} & 0 & -\dot{u}_{1} \\
-\dot{u}_{2} & \dot{u}_{1} & 0
\end{array}\right)=\left[\left(\begin{array}{ccc}
0 & -\omega & u_{2} \\
\omega & 0 & -u_{1} \\
-u_{2} & u_{1} & 0
\end{array}\right),\left(\begin{array}{ccc}
0 & 0 & u_{2} \\
0 & 0 & -u_{1} \\
-u_{2} & u_{1} & 0
\end{array}\right)\right]
$$

Proof. See [Bal1].

In general solving such optimal control problems can be difficult, but in this case one finds upon expanding the right hand side of (2.9) that $\omega(t) \equiv \omega$ (a constant). Moreover, the differential equations for $u_{1}$ and $u_{2}$ then reduce to

$$
\left(\begin{array}{l}
\dot{u}_{1} \\
\dot{u}_{2}
\end{array}\right)=\left(\begin{array}{cc}
0 & -\omega \\
\omega & 0
\end{array}\right)\left(\begin{array}{l}
u_{1} \\
u_{2}
\end{array}\right)
$$

so that the optimal control inputs take the form $u_{1}^{0}(t)=\mu \cos (\omega t+\phi), u_{2}^{0}(t)=$ $\mu \sin (\omega t+\phi)$ where

$$
\mu=\sqrt{u_{1}(0)^{2}+u_{2}(0)^{2}} \text { and } \phi=\arctan \left[\frac{u_{2}(0)}{u_{1}(0)}\right] .
$$

In light of this explicit determination of the optimal controls, the equation (2.7) may be written as

$$
\dot{X}(t)=e^{\Omega t} \Omega_{0} e^{-\Omega t} X(t), X(0)=I,
$$

where

$$
\Omega=\left(\begin{array}{ccc}
0 & -\omega & 0 \\
\omega & 0 & 0 \\
0 & 0 & 0
\end{array}\right) \text { and } \Omega_{0}=\left(\begin{array}{ccc}
0 & 0 & u_{2}(0) \\
0 & 0 & -u_{1}(0) \\
-u_{2}(0) & u_{1}(0) & 0
\end{array}\right) .
$$

Equation (2.10), in turn, may be explicitly solved for the optimal state evolution:

$$
X(t)=e^{\Omega t} e^{\left(-\Omega+\Omega_{0}\right) t}
$$

Because there is no control input directly associated with the generator $B_{3}$ of rotations about the $z$-axis, it is of particular interest to study optimal trajectories from $I$ to group elements of the form

$$
\exp \left[\left(\begin{array}{ccc}
0 & -1 & 0 \\
1 & 0 & 0 \\
0 & 0 & 0
\end{array}\right) \theta\right]=\left(\begin{array}{ccc}
\cos \theta & -\sin \theta & 0 \\
\sin \theta & \cos \theta & 0 \\
0 & 0 & 1
\end{array}\right) .
$$


To make the problem interesting, assume $\theta \neq 0(\bmod ) 2 \pi$. We use $(2.4)$ to find values of $\omega, u_{1}(0), u_{2}(0)$ such that the right hand sides of (2.11) and (2.12) are equal. We wish to solve

$$
\begin{gathered}
\left(\begin{array}{ccc}
\cos \theta & -\sin \theta & 0 \\
\sin \theta & \cos \theta & 0 \\
0 & 0 & 1
\end{array}\right)=\left(\begin{array}{ccc}
\cos \omega T & -\sin \omega T & 0 \\
\sin \omega T & \cos \omega T & 0 \\
0 & 0 & 1
\end{array}\right)\left[\left(\begin{array}{lll}
1 & 0 & 0 \\
0 & 1 & 0 \\
0 & 0 & 1
\end{array}\right)+\right. \\
\left.\left(\begin{array}{ccc}
0 & \omega^{\prime} & \omega_{2}^{\prime} \\
-\omega^{\prime} & 0 & -\omega_{1}^{\prime} \\
-\omega_{2}^{\prime} & \omega_{1}^{\prime} & 0
\end{array}\right) \sin \psi+\left(\begin{array}{ccc}
0 & \omega^{\prime} & \omega_{2}^{\prime} \\
-\omega^{\prime} & 0 & -\omega_{1}^{\prime} \\
-\omega_{2}^{\prime} & \omega_{1}^{\prime} & 0
\end{array}\right)(1-\cos \psi)\right],
\end{gathered}
$$

where

$$
\begin{gathered}
\omega^{\prime}=\omega / \sqrt{\omega^{2}+u_{1}(0)^{2}+u_{2}(0)^{2}}, \quad \omega_{i}^{\prime}=u_{i}(0) / \sqrt{\omega^{2}+u_{1}(0)^{2}+u_{2}(0)^{2}} \\
\text { and } \psi=\sqrt{\omega^{2}+u_{1}(0)^{2}+u_{2}(0)^{2}} \cdot T .
\end{gathered}
$$

Comparing matrix entries on both sides of this equation, we find that

$$
\omega \omega_{1}^{\prime}(1-\cos \psi)=\omega \omega_{2}^{\prime}(1-\cos \psi)=0 .
$$

If $\omega=0$, there could be no component of rotation about the $z$-axis. Hence we must have $\omega \neq 0$. Also, it cannot be the case that both $\omega_{i}$ 's are zero. Hence, the above equations imply that $\cos \psi=1$, so that $\psi=2 k \pi$ for some integer $k$.

In summary form, what emerges about the optimal control problem problem is the following:

(i) $\sqrt{\omega^{2}+u_{1}(0)^{2}+u_{2}(0)^{2}} \cdot T=2 k \pi$ for some integer $k$;

(ii) $\omega T=\theta+2 j \pi$ for some integer $j$;

(iii) $\sqrt{\omega^{2}+u_{1}(0)^{2}+u_{2}(0)^{2}} \cdot T=\sqrt{\mu^{2}+\omega^{2}} \cdot T$; and

(iv) the optimal cost is $\eta=\mu^{2} T=\left(u_{1}(0)^{2}+u_{2}(0)^{2}\right) T$.

By means of an elementary but detailed argument, one can show that for an optimal trajectory, $k=1$, and $j= \pm 1$, with the sign of $j$ being opposite that of $\theta$. The results of this analysis may be summarized as

TheOREM 2.2. Let $\theta \in(-\pi, \pi)$. The control inputs $u_{1}(\cdot), u_{2}(\cdot)$ that steer the system (2.7) in $T>0$ units of time from $X(0)=I$ to $X(T)=R_{z}(\theta)$ so as to minimize $\eta$ in (2.8) are of the form

$$
u_{1}^{0}(t)=\mu \cos (\omega t+\varphi), \quad u_{2}^{0}(t)=\mu \sin (\omega t+\varphi)
$$

where $\mu$ and $\varphi$ are determined by $u_{1}(0), u_{2}(0)$,

$$
\omega T= \begin{cases}\theta-2 \pi & \text { if } \theta \geq 0 \\ \theta+2 \pi & \text { if } \theta<0,\end{cases}
$$


and $u_{1}^{0}(0), u_{2}^{0}(0)$ are chosen arbitrarily subject to the constraint that

$$
u_{1}(0)^{2}+u_{2}(0)^{2}=\frac{2 \pi|\theta|-\theta^{2}}{T}
$$

The right hand quantity is also the value of $\eta / T$.

While this theorem gives a fairly complete picture of optimal trajectories from $X_{0}=I$ to $X_{1}=R_{z}(\theta)$, further remarks are in order about trajectories between arbitrary start and end points. We see that all optimal trajectories have the form (2.11), and among the choices of parameter values $\omega, u_{1}(0), u_{2}(0)$ meeting the boundary conditions, only those specified by Theorem 2.2 define optimizing trajectories between $X_{0}=I$ and $X_{1}=R_{z}(\theta)$. Note that for any choice of $\omega$ such that $-2 \pi<\omega<2 \pi$ and corresponding choices of $u_{1}(0), u_{2}(0)$ such that $\sqrt{\omega^{2}+u_{1}(0)^{2}+u_{2}(0)^{2}} T=2 \pi$, any trajectory of the form (2.11) that has initial condition $X_{0}=I$ will coincide with a $z$-axis rotation matrix $R_{z}(\theta)$ exactly once in the half-open interval $0<t \leq T$. Hence while Theorem 2.2 stipulates that to steer (2.7) optimally from $I$ to $R_{z}(\theta)$ we require $\omega \in[-2 \pi, \pi] \cup[\pi, 2 \pi)$, choices of $\omega \in(-\pi, \pi)$ with $\sqrt{\omega^{2}+u_{1}(0)^{2}+u_{2}(0)^{2}} T=2 \pi$ lead to trajectories from $I$ to some $z$-axis rotation $R_{z}(\theta)$ that are not optimal along the entire time interval although they continue to satisfy the local optimality condition (2.9). Finding the optimal evolution of (2.11) between $X_{0}=I$ and an arbitrary endpoint $X_{1} \in S O(3)$ requires consideration of all values of $\omega \in[-2 \pi, 2 \pi)$.

Example 2.3. Two-input control systems defined on $M=S^{2}$. Closely related to Example 2.2 is the following system in $\mathbb{R}^{3}$ evolving according to

$$
\dot{x}=\left(u_{1} B_{1}+u_{2} B_{2}\right) x, x(0)=x_{0} .
$$

It is easy to show that the standard 2-norm $\|x\|_{2}$ is preserved along all trajectories of this system, and hence we can think of (2.13) as a control system defined on the unit sphere $S^{2}$. We note that the vector fields $B_{1} x$ and $B_{2} x$ span the tangent space $T_{x} S^{2}$ at all points $x \in S^{2}$ except those such that the component $x_{3}=0$. Nevertheless, as noted by Brockett ([RWB0]), the system is controllable in that given any two points $x, y \in S^{2}$ and any time $T>0$ there is a pair of control inputs $u_{1}, u_{2}$ that steer (2.13) from $x$ to $y$ in $T$ units of time.

Although the state space $S^{2}$ has dimension smaller by one than the state space $S O(3)$., there is additional complexity in solving the problem of optimally steering between given endpoints so as to minimize $\eta$ in (2.8). We note that for the $S O(3)$ optimal control problem (2.7)-(2.8), the set $\left\{R_{z}(\theta):-\pi<\theta \leq \pi\right\} \subseteq S O(3)$ is a state space locus of conjugate points. That is, for any trajectory of the form (2.11) whose endpoints are $X(0)=I$ and $X(T)=R_{z}(\theta)$, one can find distinct optimal trajectories having the same endpoints and lying arbitrarily close to $X(\cdot)$ in terms of an appropriate measure of distance between trajectories. Moreover, there is an infinite 
family of optimizing trajectories from $X_{0}=I$ to $X_{1}=R_{Z}(\theta)$ that have identical cost $\eta$. This is summarized in the following.

Proposition 2.3. Suppose $u_{1}^{0}(t)=\mu \cos (\omega t+\varphi), u_{2}^{0}(t)=\mu \sin (\omega t+\varphi)$ are optimal controls that steer (2.7) from $X_{0}=I$ to $X_{1}=R_{Z}(\theta)$ so as to minimize $\eta$ in (2.8), and suppose $\alpha \in(-\pi, \pi]$. Then the control inputs $u_{1}^{*}(t)=\mu \cos (\omega t+\varphi+$ $\alpha), u_{2}^{*}(t)=\mu \sin (\omega t+\varphi+\alpha)$ also steer (2.7) from $X_{0}=I$ to $X_{1}=R_{z}(\theta)$ in the same $T$ units of time with the same value of the cost function $\eta$.

Proof. The optimal trajectory corresponding to the control inputs $u_{1}^{0}, u_{2}^{0}$ is given by (2.11) with $u_{1}(0)=\mu \cos \varphi$ and $u_{2}(0)=\mu \sin \varphi$. In terms of this $X(\cdot)$, define $Y(t)=R_{z}(\alpha) X(t) R_{z}(\alpha)^{T}$. Now $Y(0)=X(0)$ and since $R_{z}(\alpha)$ and $X_{1}=R_{z}(\theta)$ commute, it is also the case that $Y(T)=X(T)$. Now

$$
\begin{aligned}
Y(t) & =R_{z}(\alpha) e^{\Omega t} e^{\left(-\Omega+\Omega_{0}\right) t} R_{z}(\alpha)^{T} \\
& =e^{\Omega t} R_{z}(\alpha) e^{\left(-\Omega+\Omega_{0}\right) t} R_{z}(\alpha)^{T} \\
& =e^{\Omega t} e^{\left(-\Omega+\Omega_{\alpha}\right) t}
\end{aligned}
$$

where $\Omega_{\alpha}=R_{z}(\alpha) \Omega_{0} R_{z}(\alpha)^{T}$. In the same way that (2.10) and (2.11) are related, we see that $Y(\cdot)$ satisfies the differential equation

$$
\dot{Y}(t)=e^{\Omega t} \Omega_{\alpha} e^{-\Omega t} Y(t), Y(0)=I .
$$

By noting that

$$
\begin{gathered}
e^{\Omega t} \Omega_{\alpha} e^{-\Omega t}= \\
\left(\begin{array}{ccc}
0 & \mu \sin (\omega t+\alpha+\varphi) \\
0 & 0 & -\mu \cos (\omega t+\alpha+\varphi) \\
-\mu \sin (\omega t+\alpha+\varphi) & \mu \cos (\omega t+\alpha+\varphi) & 0
\end{array}\right) .
\end{gathered}
$$

we conclude the proof.

Returning to the optimal control problem of steering (2.13) between points $x_{0}$ and $x_{1}$ on $S^{2}$, we note that there is some $X \in S O(3)$ such that $x_{1}=X x_{0}$ and optimal controls steering $x_{0}$ to $x_{1}$ time $T$ are given by the solution of the corresponding problem on $S O(3)$ of steering $I$ to $X$ in one unit of time. (The proof is easy: The optimal control cost could not be greater than the least cost of steering $I$ to $Y$ over all $Y \in S O(3)$ such that $Y x_{0}=x_{1}$. Clearly, the cost could also not be less.)

This suggests that one approach to optimally steering (2.13) is to lift the problem from $S^{2}$ to $S O(3)$. Such lifting must be done carefully, however, because even if $X, Y \in$ $S O(3)$ both act on $x_{0}$ such that $X x_{0}=Y x_{0}=x_{1}$, it need not be the case that the cost (2.8) of optimally steering $I$ to $X$ is the same as the cost of steering $I$ to $Y$. On the other hand, one can also find examples of multiplicities of optimal trajectories between 
given points on $S^{2}$ that have identical costs but which look strikingly different. For example, consider the problem of steering a point on the equator of $S^{2}$ to a different point on the equator. If we impose the additional constraint that the $z$-axis orientation of the sphere should remain unchanged as a result of the motion, then the net motion is a rotation of the sphere of the form $R_{z}(\theta)$. The optimal controls are given by Theorem 2.2. By Proposition 2.3, it follows that if $u_{1}^{0}(t)=\mu \cos (\omega t+\varphi), u_{2}^{0}(t)=\mu \sin (\omega t+\varphi)$ are optimal controls steering $x_{0}$ to $x_{1}$ in this way, then for any $\psi \in[0,2 \pi)$, the control inputs $u_{1}^{*}(t)=\mu \cos (\omega t+\varphi+\psi), u_{2}^{*}(t)=\mu \sin (\omega t+\varphi+\psi)$ also steer (2.13) from $x_{0}$ to $x_{1}$ in one unit of time with the same cost $\eta$. Figure 1 illustrates two optimal paths on $S^{2}$ having the same start and end point and the same cost.

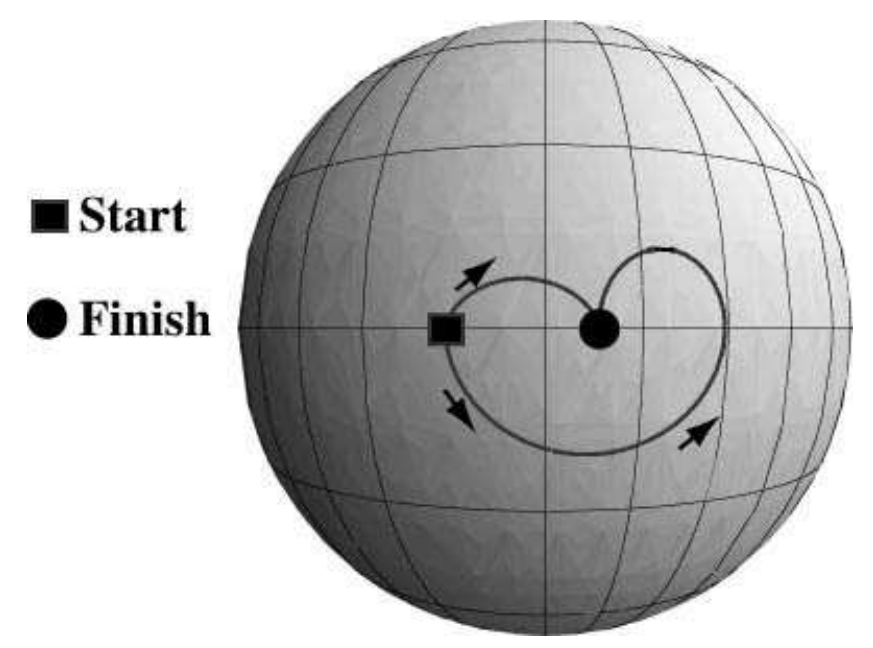

FIG. 1. As indicated in Proposition 2.3, there is an infinite family of optimizing trajectories with start and end points on the equator of $S^{2}$. The start point here (square) is $(1,0,0)$, and the end point (circle) is $(\cos (0.3), \sin (0.3), 0)$. The "clockwise" trajectory is generated by $u_{1}(t)=$ $6.27602 \cos (0.3 t+\pi), u_{2}=6.27602 \sin (0.3 t+\pi)$ while the "counterclockwise" trajectory is generated by the control inputs $u_{1}(t)=6.27602 \cos (0.3 t+0.2), u_{2}=6.27602 \sin (0.3 t+0.2)$. Both trajectories are optimizing in terms of the cost criterion (2.8), and both yield the same value of $\eta$.

\section{EXAMPLE 2.4. Models from classical and quantum mechanics.}

a) The ball and plate system: Consider a ball that rolls without slipping between two flat, horizontal plates as depicted in Figure 2. It is convenient to assume the bottom plate is fixed. Suppose the ball has unit radius, and fix a coordinate system whose $x$ - and $y$-axes lie in the fixed bottom plate with the positive $z$-axis being perpendicular to the plate in the direction of the ball. Call this the (bottom) "plate frame." We keep track of the motion of the ball by keeping track of the plate-frame coordinates of the center of the ball. We also fix an orthonormal frame in the ball, and we denote the plate-frame directions of the three coordinate axes by a $3 \times 3$ orthogonal 


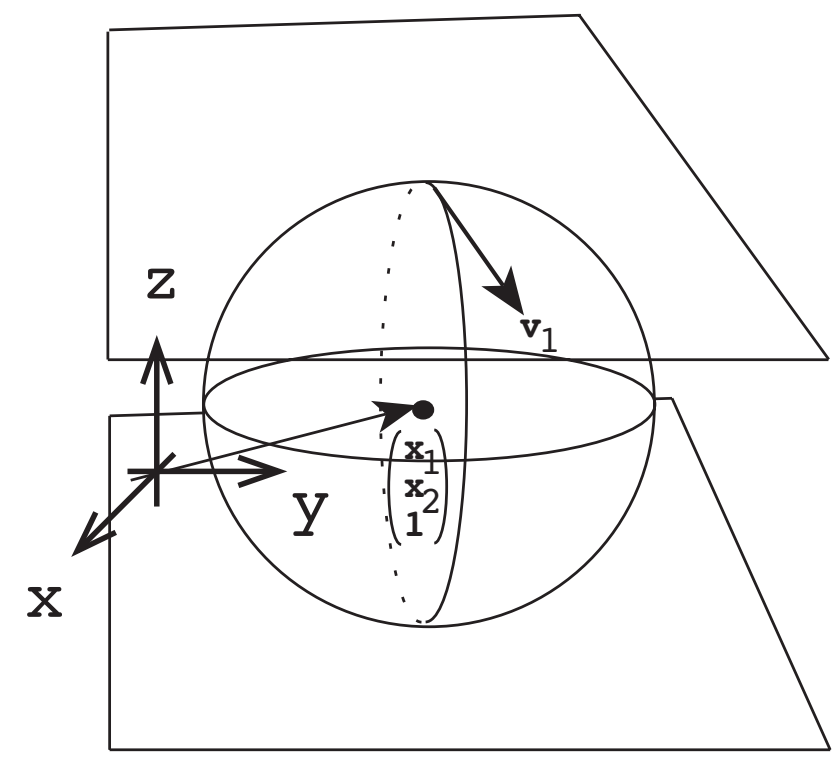

FIG. 2. A ball rolling without slipping between two flat plates. We assume the bottom plate is fixed, but the top plate moves such that it remains parallel to the bottom plate. (Figure from Baillieul and Lehman.) [Bal2].

matrix

$$
X=\left(\begin{array}{lll}
x_{11} & x_{12} & x_{13} \\
x_{21} & x_{22} & x_{23} \\
x_{31} & x_{32} & x_{33}
\end{array}\right)
$$

As the top plate moves in the plate-frame $x$-direction with velocity $v_{1}$, the center of the ball also moves in the same direction with velocity $u_{1}=v_{1} / 2$. This motion imparts a counterclockwise rotation about the $y$-axis, and since the ball has unit radius, the angular velocity is also $u_{1}$. Similarly, if the top plate moves in the (plate-frame) $y$ direction with velocity $v_{2}$, the center of the ball moves in the $y$ direction with velocity $u_{2}=v_{2} / 2$, and the angular velocity imparted about the $x$-axis is $-u_{2}$. The kinematic description of this problem is obtained by differentiating $X$ with respect to time:

$$
\dot{X}=\Omega X
$$

where

$$
\Omega=\left(\begin{array}{ccc}
0 & 0 & v_{1} / 2 \\
0 & 0 & v_{2} / 2 \\
-v_{1} / 2 & -v_{2} / 2 & 0
\end{array}\right)
$$

Note that the components of the angular velocity matrix $\Omega$ describe rotations about axes that are always parallel to the plate-frame $x$ and $y$ axes and instantaneously 
centered at the origin of the ball frame. By moving the top plate in the $x$ and $y$ directions so that $v_{1}$ and $v_{2}$ are $\pi / 2$-out-of-phase sinusoids, we can cause the ball to undergo a rotation about the $z$-axis of the type studied above.

b) Quantum spin systems: Prototypical models appearing in recent work on quantum systems describe the dynamics of spin- $1 / 2$ particles immersed in an electromagnetic field. These are modeled as systems evolving on $S U(2)$ such as (2.3) ([Dal, DAlDa]) or systems evolving on $S O(3)$ ([LiKh]). In either case, we are interested in controlled motions on the Bloch sphere, which may be thought of as a coset space $S U(2) / U(1)$ or $S O(3) / S O(2)$. In the case in which there is no Larmor dispersion, the equations of interest are

$$
\dot{x}=\epsilon\left[u_{1}(t) B_{1}+u_{2}(t) B_{2}\right] x,
$$

where $\left(u_{1}(t), u_{2}(t)\right)$ describes a time-varying rf-field that is transverse to a fixed static field aligned with the $z$-axis. The parameter $\epsilon$ models the dispersion in amplitude of the rf-field. (See $[\mathrm{LiKh}]$ for information on quantum control problems on the Bloch sphere.)

c) Two-agent control of the ball and plate system: The control objective in spin systems is to modulate the rf-field so as to influence the motion of the Bloch vector via (2.14). If we imagine the control input fields $u_{1}, u_{2}$ as generated independently-say by two agents Alice and Bob-we can think of the quantum control problem as a game where the agents must act collaboratively to produce a desired motion on the Bloch sphere. To pursue the classical mechanical analogue, suppose Alice and Bob hold adjacent sides of the (square) top plate in the above ball and plate system. Alice and Bob both push and pull the plate in sinusoidal motions of the same amplitude. By adjusting the relative phase in terms of their sinusoidal inputs, they can produce different types of motions of the Bloch vector. If the two agents execute plate motions that are perfectly in phase, the Bloch vector executes an oscillatory motion along a meridian connecting the north and south poles of the Bloch sphere. On the other hand, if Alice and Bob's sinusoids are even slightly out of phase, the motion of the vector will have a component of rotation about the vertical axis. If the sinusoids are $\pi / 2$ out-of-phase - as in the case of the optimal controls discussed above, the Bloch vector will tend to precess about the $z$-axis. Indeed, suppose the parameters $\mu, \omega$, and $\varphi$ are as in Theorem 2.2. Then applying the control inputs $u_{1}(t)=\mu \cos (\omega t+\varphi)$ and $u_{2}(t)=\mu \sin (\omega t+\varphi)$ to $(2.14)$ starting at $\left(x_{1}(0), x_{2}(0), x_{3}(0)=\left(\omega, u_{1}(0), u_{2}(0)\right)\right.$ will cause a pure precession - as depicted by the black line in Figure 3 . For initial vectors in a neighborhood of $\left(\omega, u_{1}(0), u_{2}(0)\right)$, the motion will not be a pure precession but will have an oscillatory component as also depicted in Figure 3.

REMARK 2.1. The Bloch sphere is used in quantum mechanics to represent two-state systems - known as qubits. Whether practical means of manipulating experimental realizations of qubits can be achieved is unclear. The aim of the present 


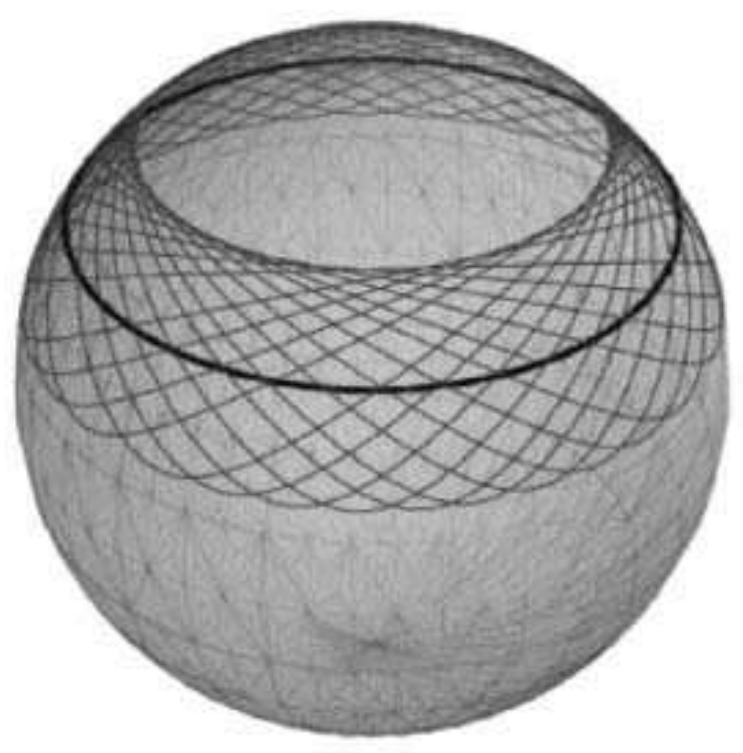

FIG. 3. Extremal paths on the Bloch sphere generated by out-of-phase sinusoidal control loops applied to (2.14).

paper is to engage in a preliminary assessment of the complexity of dealing with information by changing the state of systems of the form (2.14). It is of interest that all calculations under consideration are strictly reversible, and hence, there is no discarded information in the sense of Toffoli's discussion of reversible computations, [Tof]. The models we consider in the sequel have some but not all the features of nuclear magnetic resonance systems wherein a modulated rf-field might be used to alter the spin alignments of particles. We refer to [MaKha] for further details.

ExAmple 2.5. The Heisenberg system (also known as the nonholonomic Brockett Integrator). The systems we have defined on $S U(2), S O(3)$, and $S^{2}$ have been widely studied in both nonlinear control and mathematical physics. Another system exhibiting essential features of those above is the Heisenberg system defined on $\mathbb{R}^{3}$

$$
\left(\begin{array}{c}
\dot{x}_{1} \\
\dot{x}_{2} \\
\dot{x}_{3}
\end{array}\right)=\left(\begin{array}{c}
u_{1} \\
u_{2} \\
u_{2} x_{1}-u_{1} x_{2}
\end{array}\right) .
$$

In much of his writing over the last quarter century, R.W. Brockett chose to illustrate some fairly deep ideas regarding nonlinear geometric control theory in terms of this system. In light of this and because of its simple elegance, we shall focus the discussion in the sections to follow on the Heisenberg system. The discussion applies with little or no change to the systems introduced above. 
3. Control Communication Complexity of Distributed Control System.

In this section, we recall the concept of control communication complexity which was introduced in [Wong]. To focus on the part relevant to this paper, some simplifications and modifications have been adopted. Consider a distributed system controlled by Alice and Bob with an aim to jointly optimize the terminal state of a dynamic system

$$
(\Sigma) \quad\left\{\begin{array}{l}
\dot{x}=f\left(x, u_{A}, u_{B}\right), x_{0} \in \mathbb{R}^{N}, \\
y_{A}=h_{A}(x), \\
y_{B}=h_{B}(x) .
\end{array}\right.
$$

Here $h_{A}$ and $h_{B}$ represent the respective observation function for Alice and Bob, while $u_{A}$ and $u_{B}$ represent the respective control functions. Details on these functions will be provided below. Note that there is no direct communication link between the agents and that the initial state $x_{0}$ is assumed to be known to the agents for determining the feedback control function.

Let $\mathcal{N}_{A}$ and $\mathcal{N}_{B}$ be finite sets and $H$ be a function from $\mathcal{N}_{A} \otimes \mathcal{N}_{B} \otimes \mathbb{R}^{N}$ to $\mathbb{R}$. Given a choice of Alice, $\alpha \in \mathcal{N}_{A}$, that is not directly disclosed to Bob and a choice of Bob, $\beta \in \mathcal{N}_{B}$, that is not directly disclosed to Alice, the objective is to design corresponding control inputs $u_{A}(\alpha)$ and $u_{B}(\beta)$ that jointly drive the system (3.1) to reach a target state $x(T)$ at time $T$ so that

$$
H(\alpha, \beta, x(T))
$$

achieves the global minimal value. Moreover, it is expected that the value of $T$ and $x(T)$ should become known to the agents at some finite time $T^{\prime}$. The ordered pair, $(\Sigma, H)$ represents such a distributed control system.

In order to ensure the optimal state can be reached, the agents have to employ appropriately designed distributed controls. Following the network control models, (see for example [WoBr1], [WoBr2], [LiBa], and the references in [BaAn],) these controls are based on finitely coded observations and are communicated to the dynamical system via control codewords with finite word length. The coding and decoding are assumed to take place in discrete time intervals of length $\delta$. For simplicity, we assume that the coding/decoding intervals are of uniform duration and that $T$ is an integer multiple of $\delta$.

To fix ideas, let the observation coding functions (possibly time-dependent) be represented by $Q_{A}^{(n)}$ and $Q_{B}^{(n)}$ respectively, so that the codeword sent to Alice is represented by

$$
\psi_{A}^{(n)}=Q_{A}^{(n)}\left(h_{A}(x(n \delta))\right)
$$

The coded observation sent to Bob is represented by 


$$
\psi_{B}^{(n)}=Q_{B}^{(n)}\left(h_{B}(x(n \delta))\right)
$$

Let $\mathcal{R}_{A}^{(n)}$ and $\mathcal{R}_{B}^{(n)}$ represent the ranges of $Q_{A}^{(n)}$ and $Q_{B}^{(n)}$ respectively. These maps are assumed to be surjective and the number of information bits needed for communicating the observations to the agents is given by

$$
\left\lceil\ln _{2}\left|\mathcal{R}_{A}^{(n)}\right|\right\rceil+\left\lceil\ln _{2}\left|\mathcal{R}_{B}^{(n)}\right|\right\rceil .
$$

Note that if the coding at a given time is described by a constant function then no information bit needs to be communicated.

At time $n \delta$ the agents make decision regarding the controls to be used in the time interval

$$
[n \delta,(n+1) \delta)
$$

The choice of the control depends on, $\alpha, \beta$, and the observation codewords communicated up to the current time. One can therefore represent the control codeword sent by Alice at time $n \delta$ by

$$
\zeta_{A}^{(n)}=K_{A}^{(n)}\left(\alpha, \psi_{A}(0), \ldots, \psi_{A}(n)\right)
$$

Similarly, represent the codeword sent by Bob at time $n \delta$ is given by

$$
\zeta_{B}^{(n)}=K_{B}^{(n)}\left(\beta, \psi_{B}(0), \ldots, \psi_{B}(n)\right)
$$

Let $\mathcal{U}_{A}^{(n)}$ and $\mathcal{U}_{B}^{(n)}$ represent the set of control codewords used by Alice and Bob respectively at time $n \delta$; then the number of information bits the agents use in order to communicate their decision to the system is given by

$$
\left\lceil\ln _{2}\left|\mathcal{U}_{A}^{(n)}\right|\right\rceil+\left\lceil\ln _{2}\left|\mathcal{U}_{B}^{(n)}\right|\right\rceil
$$

The actual controls to be applied during the time interval $[n \delta,(n+1) \delta)$ are assumed to be prestored at the dynamical systems and are selected based on the sequence of control codewords received so far, so that for $t \in[n \delta,(n+1) \delta)$,

$$
u_{A}(t)=u_{A}^{(n)}\left(\zeta_{A}^{(0)}, \ldots, \zeta_{A}^{(n)}\right)(t)
$$

for Alice and

$$
u_{B}(t)=u_{B}^{(n)}\left(\zeta_{B}^{(0)}, \ldots, \zeta_{B}^{(n)}\right)(t)
$$

for Bob. 
To complete the discussion, the following definitions are modified from [Wong].

DEFINITION 3.1. The following function sequences define a protocol for the distributed control system $(\Sigma, H)$ :

$$
\text { Protocol } \Omega\left\{\begin{array}{l}
\left\{Q_{A}^{(0)}, Q_{B}^{(0)}, Q_{A}^{(1)}, Q_{B}^{(1)}, \ldots\right\}, \\
\left.K_{A}^{(0)}, K_{B}^{(0)}, K_{A}^{(1)}, K_{B}^{(1)}, \ldots\right\}, \\
\left.u_{A}^{(0)}, u_{B}^{(0)}, u_{A}^{(1)}, u_{B}^{(1)}, \ldots\right\} .
\end{array}\right.
$$

DEFinition 3.2. A protocol $\Omega$ is said to be feasible for the distributed control system $(\Sigma, H)$ if for any choices, $\alpha \in \mathcal{N}_{A}$ and $\beta \in \mathcal{N}_{B}$, there exists a finite time $T=T(\alpha, \beta)$ such that the terminal state, $x(T)$, for the dynamical system defined in (3.1) under the feedback control specified by the protocol minimizes the function $H$. Moreover, the agents can determine the value $T$ and $x(T)$ at some finite time $T^{\prime}$. The maximum of $T$ and $T^{\prime}$ is known as the termination time and in general depends on the initial choices of the agents.

If a protocol cannot achieve the target state for the choice $(\alpha, \beta)$ then $T$ is set to $\infty$.

The main difference between this distributed control problem and a centralized control problem is that Alice's control, $u_{A}(\cdot)$, cannot depend explicitly on $\beta$ and Bob's control, $u_{B}(\cdot)$, cannot depend explicitly on $\alpha$. To steer the system to the target state, the agent can start by communicating their choices to each other over the dynamical system (3.1). It may also be possible to design control laws that achieve the goal without requiring the agents to communicate their choice values to each other via the dynamical system. (How this can be done will become clear in the proof of Theorem 5.1.) Obviously, other possibilities may exist that are hybrids of these two extreme strategies. The aim of what follows is to understand the cost (in terms of say, equation (2.8), ) and complexity (as defined next) of minimizing the function $H\left(x_{0}, \cdot, \cdot\right)$ by means of (3.1).

Definition 3.3. Let $N=N(\alpha, \beta)=T(\alpha, \beta) / \delta$ be finite. The control communication complexity of $\Omega$ for the choice, $\alpha$ and $\beta$, is defined to be:

$$
c(\Omega, \alpha, \beta)=\sum_{n=0}^{N}\left\lceil\ln _{2}\left|\mathcal{R}_{A}^{(n)}\right|\right\rceil+\left\lceil\ln _{2}\left|\mathcal{R}_{B}^{(n)}\right|\right\rceil+\left\lceil\ln _{2}\left|\mathcal{U}_{A}^{(n)}\right|\right\rceil+\left\lceil\ln _{2}\left|\mathcal{U}_{B}^{(n)}\right|\right\rceil .
$$

The control communication complexity of $\Omega$ is given by

$$
C(\Omega)=\max _{\alpha, \beta} c(\Omega, \alpha, \beta) .
$$

The control communication complexity of the distributed system $(\Sigma, H)$ is defined to be the minimum of the control communication complexity over all feasible protocols:

$$
Y((\Sigma, H))=\min _{\Omega} C(\Omega) .
$$


In this formulation, termination time is not an explicit part of the complexity definition. Nevertheless, we seek to understand how the control objective affects the complexity of the information being exchanged as well as the required energy. In the models to be considered, there will be a tradeoff between the the time allowed and the energy required to achieve the control objective. Hence, the maximum value of $T$ can be regarded as another indicator of protocol complexity. This complexity will be referred to as the termination time complexity.

Both the control communication complexity and the termination time complexity do not address directly the complexity of the control function itself. Note however that there are many alternatives for defining such complexity; one approach is via the concept of "control energy":

$$
\int_{0}^{T} u_{A}^{2}(t)+u_{B}^{2}(t) d t
$$

where the integration is up to the termination time for the given choices of the agents. The maximum value of the control energy over all choices of $\alpha$ and $\beta$ made by the two agents can be regarded as a measure of the control function complexity with the understanding that controls requiring more energy are inherently more complex. This complexity will be referred to as the control energy complexity. If there is a bound $C_{\mathcal{E}}>0$ on the available input energy, then all choices must satisfy

$$
\int_{0}^{T} u_{A}^{2}(t)+u_{B}^{2}(t) d t \leq C_{\mathcal{E}}
$$

and it will not be possible to evaluate certain functions $S_{H}(\cdot, \cdot)$. For any evaluation, the control energy (3.13) required to steer systems of the type we are considering along specified paths is always inversely proportional to the time allowed, $T$.

In the rest of the paper, the relationship between control communication complexity, termination time complexity, and control energy complexity will be investigated via a concrete example, namely the Brockett Integrator.

4. Brockett Integrator and Loop Inputs. As a concrete example, consider the Heisenberg system (also known as the Brockett Integrator [RWB2]):

$$
\left(\begin{array}{c}
\dot{x} \\
\dot{y} \\
\dot{z}
\end{array}\right)=\left(\begin{array}{c}
u_{A} \\
u_{B} \\
u_{B} x-u_{A} y
\end{array}\right),\left(\begin{array}{l}
x(0) \\
y(0) \\
z(0)
\end{array}\right)=\left(\begin{array}{l}
x_{0} \\
y_{0} \\
0
\end{array}\right) \in \mathbb{R}^{3} .
$$

For observation functions, define

$$
h_{A}(t)=h_{B}(t)=z(t)
$$

Denote this system as $\Sigma_{B}$. Brockett showed in [RWB3] that for controls, $\left(u_{A}(t)\right.$, $\left.u_{B}(t)\right)$, whose trajectory, $(x(t), y(t))$, describes a simple closed curve over the control 
cycle, $[0, \tau]$, the following result holds:

$$
z(\tau)= \pm 2 A
$$

where $A$ is the area enclosed by the curve $(x(t), y(t))$. In view of this and to simplify the discussion in this paper, the control functions are assumed to satisfy the condition that the trajectory:

$$
\left(\int_{0}^{t} u_{A}(r) d r, \int_{0}^{t} u_{B}(r) d r\right)
$$

defines a closed curve as $t$ ranges from 0 to $T$. These control functions will be referred to as loop inputs.

Let $\mathcal{N}_{A}$ and $\mathcal{N}_{B}$ be finite sets. Consider a distributed control system with an optimization function, $H$, of the form

$$
H(\alpha, \beta,(x, y, z))=\left(z-S_{H}(\alpha, \beta)\right)^{2},
$$

for some function $S_{H}: \mathcal{N}_{A} \otimes \mathcal{N}_{B} \rightarrow \mathbb{R}^{3}$. Due to the loop input assumption, the terminal states are of the form:

$$
\left(x_{0}, y_{0}, z(T)\right)
$$

Hence, we can simplify the notation by assuming that $S_{H}: \mathcal{N}_{A} \otimes \mathcal{N}_{B} \rightarrow \mathbb{R}$, so that $\left(x_{0}, y_{0}, S_{H}(\alpha, \beta)\right)$ is the unique state that minimizes the function $H$ for the choice, $(\alpha, \beta)$.

As specific examples, consider the functions

$$
H_{1}(\alpha, \beta, z)=\left(z-d^{2} \alpha \beta\right)^{2},
$$

and

$$
H_{2}(\alpha, \beta, z)=\left(z+d^{2} \alpha \beta\right)^{2} .
$$

If $\mathcal{N}_{A}=\mathcal{N}_{B}=\{-1,1\}$, then the terminal states can be represented by the following tables:

\begin{tabular}{|l|c|c|}
\hline & $\beta=1$ & $\beta=-1$ \\
\hline$\alpha=1$ & $d^{2}$ & $-d^{2}$ \\
\hline$\alpha=-1$ & $-d^{2}$ & $d^{2}$ \\
\hline
\end{tabular}

Table 1

\begin{tabular}{|l|c|c|}
\hline & $\beta=1$ & $\beta=-1$ \\
\hline$\alpha=1$ & $-d^{2}$ & $d^{2}$ \\
\hline$\alpha=-1$ & $d^{2}$ & $-d^{2}$ \\
\hline
\end{tabular}

Table 2

The goal of the present section is to determine the control communication complexity of the distributed control system $\left(\Sigma_{B}, H\right)$ for several specific choices of $H$. 
For the Brockett Integrator, the control energy complexity constraint can be simplified in the following way:

$$
\begin{aligned}
C_{\mathcal{E}} & \geq \int_{0}^{T} u_{A}^{2}(t)+u_{B}^{2}(t) d t \\
& =\int_{0}^{T}\left(\frac{d x}{d t}\right)^{2}+\left(\frac{d y}{d t}\right)^{2} d t \\
& =\int_{0}^{T} \frac{d s^{2}}{d t} d t=\frac{L^{2}}{T},
\end{aligned}
$$

where $L$ is the length of the closed curve $(x(t), y(t))$. By the well-known isoperimetric inequality, (see for example [Osserman],) the following result holds:

$$
L^{2} \geq 4 \pi A
$$

Hence

$$
T C_{\mathcal{E}} \geq 4 \pi A
$$

This leads to the following result connecting termination time complexity and control energy complexity:

Proposition 4.1. For any $\alpha$ and $\beta$,

$$
2 \pi\left|S_{H}(\alpha, \beta)\right| \leq T C_{\mathcal{E}}
$$

Given a control energy constraint and any state of the form $\left(x_{0}, y_{0}, z\right)$, it is possible to design loop controls, $u_{A}$ and $u_{B}$, to reach that state if a large enough termination time is permitted. However, if the termination time is bounded, then there is a bound on the norm of the reachable state as given by (4.14).

For a general distributed control system, $\left(\Sigma_{B}, H\right)$, defined by (4.1) and (4.5), if the termination time is unconstrained, one can show that a feasible protocol always exists. The proof of this will be provided in the next section. Moreover, the control communication complexity does not depend on the control energy constraint. If the termination time is bounded, clearly each state specified in $S_{H}$ should satisfy the bound in (4.14). However, this is not a sufficient condition to guarantee the existence of a feasible protocol. These issues will be discussed in the next section.

5. Complexity of the Brockett Integrator. Consider now the distributed control system, $\left(\Sigma_{B}, H_{1}\right)$. Assume the termination time $T$ is bounded. One can construct a protocol in the following way. Depending on Alice's choice, a single bit, $\zeta_{A}^{(0)}$, set equal to either -1 or 1 is sent. Define $u_{A}$ so that for $t \in[0, T]$,

$$
u_{A}(t)= \begin{cases}-\frac{\sqrt{2 \pi} d}{T} \sin \left(\frac{2 \pi t}{T}\right) & \zeta_{A}^{(0)}=1, \\ \frac{\sqrt{2 \pi} d}{T} \sin \left(\frac{2 \pi t}{T}\right) & \zeta_{A}^{(0)}=-1 .\end{cases}
$$


Similarly, define the control function for Bob by:

$$
u_{B}(t)= \begin{cases}\frac{\sqrt{2 \pi} d}{T} \cos \left(\frac{2 \pi t}{T}\right) & \zeta_{B}^{(0)}=1, \\ -\frac{\sqrt{2 \pi} d}{T} \cos \left(\frac{2 \pi t}{T}\right) & \zeta_{B}^{(0)}=-1 .\end{cases}
$$

The trajectory of $(x(t), y(t))$ is a circle passing through the point $\left(x_{0}, y_{0}\right)$ with radius $\frac{d}{\sqrt{2 \pi}}$ and area $\frac{d^{2}}{2}$. The control energy complexity is independent of the choices of the agents and is given by

$$
2 \pi d^{2} / T
$$

Direct checking confirms that these controls realize the function $H_{1}$ and define a feasible protocol that satisfies the control energy complexity constraint, so long as

$$
2 \pi d^{2} \leq T C_{\mathcal{E}}
$$

Note that one bit is needed for each agent to signal the selected control. Moreover, since there are only two possible terminal states, the realized value can be communicated to each agent by using one bit of information at time $T$. Since two bits are needed for communicating the control decisions and two bits are needed for communication the value of $x(T)$, the control communication complexity of this protocol is four.

Similarly, under the same condition, the function $H_{2}$ can be realized by the loop inputs:

$$
\begin{aligned}
& u_{A}(t)= \begin{cases}\frac{\sqrt{2 \pi} d}{T} \sin \left(\frac{2 \pi t}{T}\right) & \zeta_{A}^{(0)}=1, \\
-\frac{\sqrt{2 \pi} d}{T} \sin \left(\frac{2 \pi t}{T}\right) & \zeta_{A}^{(0)}=-1,\end{cases} \\
& u_{B}(t)= \begin{cases}\frac{\sqrt{2 \pi} d}{T} \cos \left(\frac{2 \pi t}{T}\right) & \zeta_{B}^{(0)}=1, \\
-\frac{\sqrt{2 \pi} d}{T} \cos \left(\frac{2 \pi t}{T}\right) & \zeta_{B}^{(0)}=-1 .\end{cases}
\end{aligned}
$$

The control energy complexity of $H_{2}$ is identical to $H_{1}$ and is given by

$$
2 \pi d^{2} / T \text {. }
$$

Consider now the function $H_{3}$, which is defined as:

$$
H_{3}(\alpha, \beta, z)=\left(z-\frac{d^{2}}{2}[\beta(\beta-1)+\alpha \beta(\beta+1)]\right)^{2} .
$$

Taking loop inputs of the above form, the corresponding optimal state $S_{H}(\alpha, \beta)$ of this function is given by the following table:

\begin{tabular}{|l|c|c|}
\hline & $\beta=1$ & $\beta=-1$ \\
\hline$\alpha=1$ & $d^{2}$ & $d^{2}$ \\
\hline$\alpha=-1$ & $d^{2}$ & $-d^{2}$ \\
\hline
\end{tabular}

Table 3 
Note that the norm of the states in Table 3 is equal to that of Table 1 and 2 . However, if

$$
d^{2}=\frac{T C_{\mathcal{E}}}{2 \pi},
$$

then there is no feasible protocol that can satisfy the control energy complexity constraint (3.14). To prove this note that the left hand side of equation (5.9) can be written as

$$
-2 \int_{0}^{T} y d x=2 A
$$

for trajectories that are closed smooth curves, while the right hand side is lower bounded by $L^{2} /(2 \pi)$. Hence equation (5.9) implies:

$$
4 \pi A=L^{2} .
$$

It is known that the isoperimetric inequality holds for general smooth closed curves (that is, smooth closed curves which may not be simple), with the equality holding only for circles. (See Lemma 1.2 of [Osserman].) Let $u_{A}^{(\alpha, \beta)}$ be the control function used by Alice and $u_{B}^{(\alpha, \beta)}$ be the function used by Bob as prescribed by the protocol. It follows that as $t$ ranges from 0 to $T$, the trajectory $\left(u_{A}^{(\alpha, \beta)}(t), u_{B}^{(\alpha, \beta)}(t)\right)$ describes a circle with radius $\frac{\sqrt{T C_{\mathcal{E}}}}{2 \pi}$ of the form:

$$
\left(\frac{\sqrt{T C_{\mathcal{E}}}}{2 \pi} \cos \left(\frac{2 \pi t}{T}+\phi\right), \frac{\sqrt{T C_{\mathcal{E}}}}{2 \pi} \sin \left(\frac{2 \pi t}{T}+\phi\right)\right)
$$

for some phase value $\phi$. Since Bob is not aware of Alice's choice, $\alpha$, at time zero, there is a time $\tau>0$, such that

$$
u_{B}^{(d, \beta)}(t)=u_{B}^{(-d, \beta)}(t)
$$

for $t \in[0, \tau)$. It follows that this must hold for all $t$ and

$$
u_{B}^{(d, \beta)}=u_{B}^{(-d, \beta)} .
$$

Similar argument shows that

$$
u_{A}^{(\alpha, d)}=u_{A}^{(\alpha,-d)}
$$

The functions that can be realized are $H_{1}$ and $H_{2}$ under the stated control energy constraints, but not $\mathrm{H}_{3}$.

If

$$
d^{2}<\frac{T C_{\mathcal{E}}}{2 \pi}
$$


then there can be more than one way to construct a feasible protocol for $H_{3}$. One approach is provided by Theorem 5.1. We consider a different approach here. Let $\delta>0$ be chosen to satisfy:

$$
d^{2}<\frac{(T-\delta) C_{\mathcal{E}}}{2 \pi}
$$

The basic idea is to construct a protocol with two phases. In the first phase, lasting from 0 to $\delta$, one of the agents, say Alice, communicates her choice to the other agent. This can be done, for example, by setting

$$
\begin{aligned}
& u_{A}(t)=\epsilon \operatorname{sgn}(\alpha) \sin \left(\frac{2 \pi t}{\delta}\right), \\
& u_{B}(t)=\epsilon \cos \left(\frac{2 \pi t}{\delta}\right),
\end{aligned}
$$

for $t \in[0, \delta)$. Note that Bob's control can be pre-determined as it does not depend on $\beta$, and hence requires no control bit for communication. The value of $\alpha$ can be decided from reading the sign of $z(\delta)$. By setting $\epsilon$ to be close to zero, the control energy in this phase can be arbitrarily small. In the second phase, lasting from $\delta$ to $T$, Alice sets the control function to be

$$
u_{A}(t)=\frac{\sqrt{2 \pi} d}{T-\delta} \sin \left(\frac{2 \pi(t-\delta)}{T-\delta}\right),
$$

while the control function for Bob is of the form:

$$
u_{B}(t)= \pm \frac{\sqrt{2 \pi} d}{T-\delta} \cos \left(\frac{2 \pi(t-\delta)}{T-\delta}\right)
$$

The sign for $u_{B}$ is chosen based on $(\alpha, \beta)$, the value of which is known to Bob at time $\delta$, after having received the information bit indicating the sign of $z(\delta)$. On the other hand, the control of Alice is pre-determined as it does not depend on $\alpha$, and hence requires no control bit for communication. At time $T$, Alice can be informed of the terminal state value by one bit of information. Therefore, this protocol requires four bits of communication information: one control bit and one observation bit for Alice and similarly for Bob.

One can summarize these results in the following proposition:

Proposition 5.1. If

$$
2 \pi d^{2} \leq T C_{\mathcal{E}}
$$

the distributed control system $\left(\Sigma_{B}, H_{1}\right)$ or $\left(\Sigma_{B}, H_{2}\right)$ has a control communication complexity equal to four under the control energy constraint (3.14). There is no feasible protocol otherwise. For the distributed control system, $\left(\Sigma_{B}, H_{3}\right)$, if

$$
2 \pi d^{2}<T C_{\mathcal{E}}
$$

the control communication complexity is four. Otherwise there is no feasible protocol. 
Proof. We have shown that there exist protocols for these functions that require four bits of communication information. If follows from Theorem 1 of [Wong] that the lower bound of the control communication complexity of these systems is four. Hence the control communication complexity is four. The other statements are established by the discussion preceding the statement of the proposition.

For the case where time complexity is not constrained, the following result holds:

TheOREM 5.1. Let $H$ be a function from $\mathcal{N}_{A} \otimes \mathcal{N}_{B} \otimes \mathbb{R}$ to $\mathbb{R}$ where $\left|\mathcal{N}_{A}\right|=N_{A}$ and $\left|\mathcal{N}_{B}\right|=N_{B}$. Then the control communication complexity of the distributed control system, $\left(\Sigma_{B}, H\right)$, is bounded above by:

$$
\left\lceil\ln _{2} N_{A}\right\rceil+\left\lceil\ln _{2} N_{B}\right\rceil
$$

Proof. Represent the choices of Alice by $\{1,2, \ldots, I\}$ and the choices of Bob by $\{1,2, \ldots, J\}$. For any positive $\tau$, set the terminating time, $T$, to be

$$
T=I \tau
$$

Define a protocol so that when Alice chooses $i$, the corresponding control for Alice is of the form:

$$
u_{i}(t)= \begin{cases}-\frac{\sqrt{2 \pi}}{\tau} \sin \left(\frac{2 \pi t}{\tau}\right) & t \in[(i-1) \tau, i \tau), \\ 0 & \text { otherwise. }\end{cases}
$$

If Bob chooses $j$, then the corresponding control for Bob is of the form:

$$
v_{j}(t)= \begin{cases}\frac{\sqrt{2 \pi}}{\tau} H(1, j) \cos \left(\frac{2 \pi t}{\tau}\right) & t \in[0, \tau), \\ \frac{\sqrt{2 \pi}}{\tau} H(2, j) \cos \left(\frac{2 \pi t}{\tau}\right) & t \in[\tau, 2 \tau), \\ \vdots & \vdots \\ \frac{\sqrt{2 \pi}}{\tau} H(I, j) \cos \left(\frac{2 \pi t}{\tau}\right) & t \in[(I-1) \tau, I \tau] .\end{cases}
$$

One can show that if $u_{i}$ and $v_{j}$ are used, then $z(T)=H(i, j)$. Moreover,

$$
\int_{0}^{T} u_{i}^{2}(t)+v_{j}^{2}(t) d t=\frac{\pi I^{2}}{T}\left(1+\sum_{k=1}^{I} H^{2}(k, j) .\right)
$$

By choosing $\tau$ to be large enough, one can see that the control energy complexity constraint can be satisfied.

The protocol described in the proof to the previous theorem has a control energy complexity equal to:

$$
\left(4+2 / d^{2}\right) \frac{2 \pi d^{2}}{T},
$$

which is $\left(4+2 / d^{2}\right)$ times larger than the minimum control energy for $H_{1}$ or $H_{2}$. When $T$ is required to be fixed or bounded, but if $d$ is small enough, then this provides an alternative protocol for solving the distributed control system, $\left(\Sigma_{B}, H_{3}\right)$. At the 
terminal time, the agents need to be informed of the terminal state value. Hence the communication control complexity of this protocol is also four.

If the loop inputs are restricted to be piecewise constant functions, the trajectory of $(x(t), y(t))$ is a $k$-sided polygon if it is a closed curve. In this case, the isoperomertric inequality is sharpened to [Osserman]:

$$
\frac{L^{2}}{A} \geq \frac{4}{k} \tan \frac{\pi}{k}>4 \pi
$$

From this one can obtain the following result.

Proposition 5.2. Consider the distributed control system, $\left(\Sigma_{B}, H_{1}\right)$ or $\left(\Sigma_{B}\right.$, $\left.\mathrm{H}_{2}\right)$, using piecewise constant controls with a trajectory $(x(t), y(t))$ defining a $k$-side polygon. Under the control energy constraint (3.14) there is no feasible protocol if

$$
d^{2} \frac{2}{k} \tan \frac{\pi}{k}>T C_{\mathcal{E}}
$$

Otherwise, the control communication complexity is four.

We conclude the section with remarks on the control energy complexity of the distributed control system, $\left(\Sigma_{B}, H_{3}\right)$. From previous discussion we know that there exists a feasible protocol that requires two communication bits and has a control energy complexity that is $\left(4+2 / d^{2}\right)$ times larger than that of either $\left(\Sigma_{B}, H_{1}\right)$ or $\left(\Sigma_{B}, H_{2}\right)$. We will show in the following example how to construct lower control energy protocols that require the same amount of communication bits.

5.1. The lazy Bob protocol. If Alice and Bob are allowed to each execute two loops instead of one, the following inputs define a protocol solving $\left(\Sigma_{B}, H_{3}\right)$. Alice executes

$$
u_{A}(t)=a \sin \frac{4 \pi t}{T} \quad 0 \leq t \leq T
$$

in the case that $\alpha=1$, and

$$
u_{A}(t)=\left\{\begin{array}{cl}
a \sin \frac{4 \pi t}{T} & 0 \leq t \leq T / 2 \\
-a \sin \frac{4 \pi t}{T} & T / 2<t \leq T
\end{array}\right.
$$

in the case that $\alpha=-1$. Represent the double loop control in (5.31) as $(a, a)$ and the double loop control in (5.32) as $(a,-a)$.

Bob, on the other hand, executes only a single loop - either in the interval $[0, T / 2]$ if $\beta=1$ or in $[T / 2, T]$ if $\beta=-1$. Bob provides zero input during half the time interval $[0, T]$ in either case. (Hence the name of the protocol.) Represent Bob's control loop by:

$$
\begin{aligned}
& (b, 0) \quad \leftrightarrow \quad u_{B}(t)=\left\{\begin{array}{cc}
b \cos \frac{4 \pi t}{T} & 0 \leq t \leq T / 2 \\
0 & T / 2<t \leq T ;
\end{array}\right. \\
& (0, b) \quad \leftrightarrow \quad u_{B}(t)=\left\{\begin{array}{cc}
0 & 0 \leq t \leq T / 2 \\
b \cos \frac{4 \pi t}{T} & T / 2<t \leq T .
\end{array}\right.
\end{aligned}
$$


Taking these inputs, the state table $S_{H}(\alpha, \beta)$ (corresponding to (5.8)) is

\begin{tabular}{|l|c|c|}
\hline & $(b, 0)$ & $(0, b)$ \\
\hline$(a, a)$ & $-\frac{a b T^{2}}{8 \pi}$ & $-\frac{a b T^{2}}{8 \pi}$ \\
\hline$(a,-a)$ & $-\frac{a b T^{2}}{8 \pi}$ & $\frac{a b T^{2}}{8 \pi}$ \\
\hline
\end{tabular}

Table 4

For consistency with Table 3 , the terms $a$ and $b$ are chosen so that

$$
-\frac{a b T^{2}}{8 \pi}=d^{2}
$$

We note that the cost of evaluating any cell in Table 4 is

$$
\int_{0}^{T} u_{A}(t)^{2}+u_{B}(t)^{2} d t=\frac{1}{2}\left(a^{2}+\frac{b^{2}}{2}\right) T .
$$

From this, an easy calculation shows that the loop amplitudes that minimize this cost subject to the constraint (5.33) are

$$
a= \pm 2 \cdot 2^{\frac{1}{4}} \frac{\sqrt{\pi} d}{T}, \quad b=\mp 2 \cdot 2^{\frac{3}{4}} \frac{\sqrt{\pi} d}{T}
$$

with corresponding cost $\frac{2 \pi 2 \sqrt{2} d^{2}}{T}$. For the sake of comparison, the cost (=control energy (3.14)) of computing entries for $H_{1}$ and $H_{2}$ in Tables 1,2 resp. is $\frac{2 \pi d^{2}}{T}$, showing the cost of the Lazy Bob protocol to be higher by a factor of $2 \sqrt{2}$.

Assuming the absolute value of the function $\mathrm{H}$ is fixed to be $d^{2}$, there are a total of sixteen $2 \times 2$ matrices which can be listed explicitly as

$$
d^{2}\left(\begin{array}{ll}
\sigma_{11} & \sigma_{12} \\
\sigma_{21} & \sigma_{22}
\end{array}\right)
$$

where $\sigma_{i j}= \pm 1$. There are clearly sixteen such matrices, and these can be divided into two groups of eight. All those having an odd number of $\sigma_{i j}$ 's equal to -1 have rank 2. All those having an even number of $\sigma_{i j}$ 's equal to -1 have rank 1.

Proposition 5.1 can be extended to all these cases, so that all functions with the same rank have identical complexity. This phenomenon is not a coincidence as the analysis in ([WongBal] $)$ reveals.

\section{Phased-Loop Protocols and Parametrically Optimal Solutions to the}

Standard Parts Problem. Suppose Alice chooses the control loop input

$$
u_{A}(t)=a \sin \left(\frac{2 \pi t}{T}+\varphi_{A}\right),
$$

and Bob chooses

$$
u_{B}(t)=b \sin \left(\frac{2 \pi t}{T}+\varphi_{B}\right)
$$


Then in $T$ units of time, the system (4.1) evolves from $(x(0), y(0), z(0))=(0,0,0)$ to $(0,0, z(T))$ where

$$
z(T)=\frac{a b T^{2}}{2 \pi} \sin \left(\varphi_{A}-\varphi_{B}\right)
$$

\begin{tabular}{|l|c|c|}
\hline & $\zeta_{B}^{(0)}=1$ & $\zeta_{B}^{(0)}=0$ \\
\hline$\zeta_{A}^{(0)}=1$ & $d^{2}$ & $d^{2}$ \\
\hline$\zeta_{A}^{(0)}=0$ & $d^{2}$ & $-d^{2}$ \\
\hline
\end{tabular}

Table 5

We consider a restricted version of the standard parts optimization problem introduced in Section 1. The problem is to have Alice and Bob compute the function specified in Table 5 using the following choices of input loops: Suppose we define

$$
\left(\begin{array}{c}
\text { Alice } \\
\text { loops }
\end{array}\right)=\left\{\begin{array}{lll}
u_{A 1}(t)=a_{1} \sin \left(\frac{2 \pi t}{T}-\varphi_{1}\right) & 0 \leq t \leq T & \text { if } \quad \zeta_{A}^{(0)}=1 \\
u_{A 2}(t)=a_{2} \sin \left(\frac{2 \pi t}{T}-\varphi_{2}\right) & 0 \leq t \leq T & \text { if } \quad \zeta_{A}^{(0)}=0
\end{array}\right.
$$

and

$$
\left(\begin{array}{c}
\text { Bob } \\
\text { loops }
\end{array}\right)=\left\{\begin{array}{lll}
u_{B 1}(t)=b_{1} \sin \left(\frac{2 \pi t}{T}-\psi_{1}\right) & 0 \leq t \leq T & \text { if } \quad \zeta_{B}^{(0)}=1 \\
u_{B 2}(t)=b_{2} \sin \left(\frac{2 \pi t}{T}-\psi_{2}\right) & 0 \leq t \leq T & \text { if } \quad \zeta_{B}^{(0)}=0
\end{array}\right.
$$

The goal of the optimization is to select values of the parameters $a_{i}, b_{j}, \varphi_{k}, \psi_{\ell}, 1 \leq$ $i, j, k, \ell \leq 2$ such that

$$
\eta=\int_{0}^{T} u_{A 1}(t)^{2}+u_{A 2}(t)^{2}+u_{B 1}(t)^{2}+u_{B 2}(t)^{2} d t
$$

is minimized. This is a restricted version of the standard parts problem in the sense that the functional is to be minimized with respect to the parameters and over an infinite-dimensional function space of control inputs. The restriction simplifies the problem in several ways. First the integral can be easily evaluated, and in light of (6.3), the optimization problem reduces to finding the minimum of

$$
a_{1}^{2}+a_{2}^{2}+b_{1}^{2}+b_{2}^{2}
$$

subject to the following table of constraints: 


\begin{tabular}{|l|c|c|}
\hline & $\zeta_{B}^{(0)}=1$ & $\zeta_{B}^{(0)}=0$ \\
\hline$\zeta_{A}^{(0)}=1$ & $a_{1} b_{1} \sin \left(\varphi_{1}-\psi_{1}\right)=\bar{d}^{2}$ & $a_{1} b_{2} \sin \left(\varphi_{1}-\psi_{2}\right)=\bar{d}^{2}$ \\
\hline$\zeta_{A}^{(0)}=0$ & $a_{2} b_{2} \sin \left(\varphi_{2}-\psi_{1}\right)=\bar{d}^{2}$ & $a_{2} b_{2} \sin \left(\varphi_{2}-\psi_{2}\right)=-\bar{d}^{2}$ \\
\hline \multicolumn{2}{|l|}{ where $\bar{d}=d \sqrt{2 \pi} / T$ with $d$ as given in Table 5. } \\
\hline
\end{tabular}

Table $5^{\prime}$

Each entry in the table depends only on the phase differences $\varphi_{i}-\psi_{j}(i, j=1,2)$, and hence there is no loss of generality in assuming that $\varphi_{1}=0$. Using the constraints in the table, we can rewrite the objective function (6.5) in terms of $a_{1}$ to get

$$
a_{1}^{2}\left(1+\lambda^{2}\right)+\frac{1}{a_{1}^{2}} \mu^{2}
$$

where

$$
\lambda=-\frac{\sin \psi_{1}}{\sin \left(\varphi_{2}-\psi_{1}\right)} \quad \text { and } \quad \mu=\bar{d} \sqrt{\frac{1}{\sin ^{2} \psi_{1}}+\frac{1}{\sin ^{2} \psi_{2}}} .
$$

This is easily seen to be minimized with respect to $a_{1}$ if

$$
a_{1}= \pm\left(\frac{\mu^{2}}{1+\lambda^{2}}\right)^{\frac{1}{4}} \text {. }
$$

The corresponding value of the objective function is $2 \sqrt{\mu^{2}\left(1+\lambda^{2}\right)}$, which in terms of the phase parameters may be written as

$$
2 \bar{d} \frac{\sqrt{\left(\sin ^{2} \psi_{1}+\sin ^{2} \psi_{2}\right)\left(\sin ^{2} \psi_{1}+\sin ^{2}\left(\varphi_{2}-\psi_{1}\right)\right)}}{\left|\sin \psi_{1} \sin \psi_{2} \sin \left(\varphi_{2}-\psi_{1}\right)\right|} .
$$

The constraints of Table $5^{\prime}$ allow us to express the amplitude parameters $a_{2}, b_{1}, b_{2}$ in terms of the phase parameters $\varphi_{2}, \psi_{1}, \psi_{2}$, and moreover, they impose a relationship among these parameters given by

$$
\sin \psi_{1} \sin \left(\varphi_{2}-\psi_{2}\right)+\sin \psi_{2} \sin \left(\varphi_{2}-\psi_{1}\right) .
$$

This defines $\varphi_{2}$ as an implicit function of $\psi_{1}$ and $\psi_{2}$ which may be written explicitly as

$$
\begin{aligned}
& \varphi_{2}\left(\psi_{1}, \psi_{2}\right)=\arctan \left[2 \sin \psi_{1} \sin \psi_{2}, \sin \left(\psi_{1}+\psi_{2}\right)\right] \text { or } \\
& \varphi_{2}\left(\psi_{1}, \psi_{2}\right)=\arctan \left[-2 \sin \psi_{1} \sin \psi_{2},-\sin \left(\psi_{1}+\psi_{2}\right] .\right.
\end{aligned}
$$

The values of $\varphi_{2}$ in these formulas differ by $\pi$, but the minimum values of the expression (6.6) are the same in either case. Using elementary techniques involving first and second derivatives, we find that in the range $-\pi<\psi_{1}, \psi_{2}<\pi$, (6.6) has local 
minima at the eight points $(\pi / 4,-\pi / 4),(-\pi / 4, \pi / 4),(3 \pi / 4,-3 \pi / 4),(-3 \pi / 4,3 \pi / 4)$, $(\pi / 4,3 \pi / 4),(\pi / 4,-3 \pi / 4),(3 \pi / 4, \pi / 4),(-3 \pi / 4,-\pi / 4)$. The function (6.6) takes on the same value for each of these choices. For any of these minima, the corresponding value of $\varphi_{2}$ may be selected as $\pm \pi / 2$, and for either of these values, we may find corresponding values of the amplitude parameters $a_{i}, b_{j}$ from Table $5^{\prime}$. The signs of the these parameters must be chosen to satisfy the constraints of Table $5^{\prime}$, but in all cases the magnitude is $2^{\frac{1}{4}} \bar{d}=2^{\frac{1}{4}} \sqrt{2 \pi} d / T$. The corresponding optimal value of the objective function (6.5) is $\frac{8 \pi \sqrt{2} d^{2}}{T^{2}}$ with the corresponding value of $\eta$ given by $\frac{4 \pi \sqrt{2} d^{2}}{T}$. We summarize these remarks in the following:

Proposition 6.1. The problem of finding control inputs $\left(u_{A i}, u_{B j}\right)$ that steer the system (4.1) so as to evaluate the function given in Table 5 is solved by the functions (Alice loops) and (Bob loops) given above, where we specify that $\left|\varphi_{1}-\varphi_{2}\right|=\pi / 2$, $\left|\psi_{1}-\psi_{2}\right|=\pi / 2$, and $\left|\varphi_{1}-\psi_{1}\right|=\pi / 4$ or $3 \pi / 4$, and all amplitude parameters $a_{i}, b_{j}$ will have equal magnitudes and can be specified as

$$
\begin{array}{ll}
a_{1}=2^{\frac{1}{4}} \sqrt{2 \pi} d / T, & a_{2}=\operatorname{sgn}\left[\sin \left(\varphi_{1}-\psi_{1}\right) \sin \left(\varphi_{2}-\psi_{1}\right)\right] a_{1} \\
b_{1}=\operatorname{sgn}\left[\sin \left(\varphi_{1}-\psi_{1}\right)\right] a_{1}, & b_{2}=\operatorname{sgn}\left[\sin \left(\varphi_{1}-\psi_{2}\right)\right] a_{1} .
\end{array}
$$

Among all sinusoidal inputs depending on phase and amplitude parameters as in (6.1) and (6.2), these choices locally minimize the performance index (6.4).

REMARK 6.1. The optimal cost of the solution to the standard parts problem given by Proposition 6.1 is of course directly related to the energy complexity as discussed in the previous two sections. To make a direct comparison with our previous calculations, we note that the energy complexity will be half the optimal standard parts value -i.e. $\frac{2 \pi \sqrt{2} d^{2}}{T}$. Thus the complexity is half that of the "Lazy Bob" protocol but a factor of $\sqrt{2}$ larger than the energy complexity of the solutions (5.1)-(5.2) and (5.5)-(5.6) for the functions given in Tables 1,2 in Section 5. It follows that the Lazy Bob Protocol is not optimal.

REMARK 6.2. A geometric variational solution to the problem of finding input pairs $\left(u_{A i}, u_{B j}\right)$ that compute the function of Table 5 while minimizing (6.4) over the set of all functions in, say, $L_{2}[0, T]$ is not presently available. It will be shown elsewhere ([WongBa1]) in the context of a broader theory that the phased-loop solution presented here has the same minimum energy requirement as that of a different set of parametrically-optimal solutions in which inputs are represented by Fourier series on $[0, T]$. While the phased-loop solution to the minimum-energy evaluation of $2 \times 2$ matrices appears to be a natural extension of classical results in geometric optimal control, further work must be done to characterize the matrices that can be evaluated in this way. This work will be reported elsewhere.

7. The Effect of Phase Uncertainty in the Input Loops. In thinking about physical implementations of the above distributed control systems - for instance in quantum spin systems or in our ball-and-plate system from classical mechanics, we 
turn our attention to the phase relationships of the two control input loops. To fix ideas, consider two control inputs $u_{A}$ and $u_{B}$ to (4.1) which generate $x$ and $y$ trajectories:

$$
x(t)=\lambda \sin (\omega t+\varphi) \text { and } y(t)=\lambda \sin (\omega t+\varphi+\psi) .
$$

We explicitly integrate $\dot{z}=u_{B} x-u_{A} y$ to obtain

$$
z(t)=z_{0}-\left(\lambda^{2} \omega \sin \psi\right) t
$$

Over one cycle, $0 \leq t \leq 2 \pi / \omega,(x(t), y(t))$ traces an ellipse whose semi-major axes are aligned with the positive and negative forty-five degree lines in the $x, y$-plane. In agreement with (4.3), the change in the value of $z$ over this cycle, $2 \pi \lambda^{2} \sin \psi$, is two times the area of the ellipse. Thus, the phase offset of the control loop inputs $u_{A}(\cdot)$ and $u_{B}(\cdot)$ is seen to be a geometric quantity.

To explore the geometric phase in more detail, we note that the control inputs $u_{A}, u_{B}$ that generate the elliptical path in the $x, y$-plane are given by

$$
u_{A}(t)=\lambda \omega \cos (\omega t+\varphi) \text { and } u_{B}(t)=\lambda \omega \cos (\omega t+\varphi+\psi) .
$$

A straightforward calculation shows that these satisfy the ordinary differential equation:

$$
\left(\begin{array}{c}
\dot{u}_{A} \\
\dot{u}_{B}
\end{array}\right)=\left(\begin{array}{ll}
-\alpha & \beta \\
-\beta & \alpha
\end{array}\right)\left(\begin{array}{l}
u_{A} \\
u_{B}
\end{array}\right)
$$

where $\alpha=\omega \cos \psi / \sin \psi$ and $\beta=\omega / \sin \psi$. This representation allows us to examine the geometric aspect of phase differences in the inputs in the context of the $S U(2), S O(3)$, and $S^{2}$ examples of Section 2. To fix ideas we return to Examples 2.2 and 2.3. By a slightly inexact analogy with equation (2.9), it follows from (7.1) that we can write

$$
\dot{\Omega}=A(\psi) \omega \Omega+\Omega A(\psi)^{T} \omega
$$

where

$$
\Omega(t)=\left(\begin{array}{ccc}
0 & 0 & u_{B}(t) \\
0 & 0 & -u_{A}(t) \\
-u_{B}(t) & u_{A}(t) & 0
\end{array}\right)
$$

and

$$
A(\psi)=\left(\begin{array}{ccc}
\frac{\cos \psi}{\sin \psi} & \frac{1}{\sin \psi} & 0) \\
-\frac{1}{\sin \psi} & -\frac{\cos \psi}{\sin \psi} & 0 \\
0 & 0 & 0
\end{array}\right) .
$$


Equation (7.2) may be explicitly solved, and we write the solution as

$$
\Omega(t)=e^{A(\psi) \omega t} \Omega_{0} e^{A(\psi)^{T} \omega t},
$$

where

$$
\Omega_{0}=\left(\begin{array}{ccc}
0 & 0 & u_{B}(0) \\
0 & 0 & -u_{A}(0) \\
-u_{B}(0) & u_{A}(0) & 0
\end{array}\right)
$$

A subtle but crucial difference between

$$
\dot{X}(t)=\Omega(t) X(t), \quad X(0)=I
$$

and equation (2.10) is that the exponentiated $A(\psi) \neq-A(\psi)^{T}$, and we do not have an elegantly simple solution to (7.4) along the lines of (2.11). We therefore turn to approximation for further insight. Specifically, we consider small deviations from the ideal case of the previous sections in which $u_{A}(\cdot)$ and $u_{B}(\cdot)$ are sinusoids whose phases differ by $90^{\circ}$-i.e. $\psi= \pm \pi / 2$. Consider specifically the case $\psi=-\pi / 2+\epsilon$, so that

$$
A\left(\frac{\pi}{2}+\epsilon\right)=B+\epsilon C+o(\epsilon)
$$

where

$$
B=\left(\begin{array}{ccc}
0 & -1 & 0 \\
1 & 0 & 0 \\
0 & 0 & 0
\end{array}\right) \text { and } C=\left(\begin{array}{ccc}
-1 & 0 & 0 \\
0 & 1 & 0 \\
0 & 0 & 0
\end{array}\right)
$$

Noting that $B C+C B=0$, a straightforward calculation shows that

$$
e^{A\left(\frac{\pi}{2}+\epsilon\right) \omega t}=e^{B \omega t}+\epsilon C \sin (\omega t)+o(\epsilon) .
$$

We can prove the following:

LEMma 7.1. Consider the control system (2.7) where the control inputs are

$$
\begin{aligned}
& u_{1}(t)=\lambda \omega \cos (\omega t+\varphi), \\
& u_{2}(t)=\lambda \omega \sin (\omega t+\varphi+\epsilon),
\end{aligned}
$$

with $\epsilon$ being a small positive quantity. The equation (2.7) may be written as

$$
\dot{X}_{\epsilon}(t)=\Omega_{\epsilon}(t) X_{\epsilon}(t), \quad X(0)=I,
$$

where

$$
\Omega_{\epsilon}(t)=e^{B \omega t} \Omega_{0} e^{-B \omega t}+\epsilon \sin \omega t\left(C \Omega_{0} e^{-B \omega t}+e^{B \omega t} \Omega_{0} C\right)+o(\epsilon)
$$

and $B$ and $C$ are as above. 
Proof. If we specify $\psi=\frac{\pi}{2}+\epsilon$ in the above definition of $u_{B}$ and then rename $u_{A}$ to be $u_{1}$ and $u_{B}$ to be $u_{2}$, it is obvious that

$$
\left(\begin{array}{c}
\dot{u}_{1} \\
\dot{u}_{2}
\end{array}\right)=\left(\begin{array}{cc}
-\alpha & \beta \\
-\beta & \alpha
\end{array}\right)\left(\begin{array}{l}
u_{1} \\
u_{2}
\end{array}\right),
$$

with $\alpha, \beta$ being defined as in equation (7.1). Expanding these coefficients along the lines of (7.5), we write

$$
\alpha=\omega \epsilon+o(\epsilon) \text { and } \beta=-\omega+o(\epsilon) .
$$

Then (7.9) may be rewritten as

$$
\left(\begin{array}{l}
\dot{u}_{1} \\
\dot{u}_{2}
\end{array}\right)=\left[\left(\begin{array}{cc}
0 & -\omega \\
\omega & 0
\end{array}\right)+\left(\begin{array}{cc}
-\epsilon \omega & 0 \\
0 & \epsilon \omega
\end{array}\right)+o(\epsilon)\right]\left(\begin{array}{l}
u_{1} \\
u_{2}
\end{array}\right) .
$$

From this, it follows that

$$
\left(\begin{array}{l}
u_{1}(t) \\
u_{2}(t)
\end{array}\right)=e^{(\bar{B}+\epsilon \bar{C}) \omega t}\left(\begin{array}{l}
u_{1}(0) \\
u_{2}(0)
\end{array}\right)+o(\epsilon),
$$

where

$$
\bar{B}=\left(\begin{array}{cc}
0 & -1 \\
1 & 0
\end{array}\right) \text { and } \bar{C}=\left(\begin{array}{cc}
-1 & 0 \\
0 & 1
\end{array}\right) .
$$

To render the zeroth and first-order terms explicitly, we expand $e^{(\bar{B}+\epsilon \bar{C}) \omega t}$ as a series, and as above, we note that $\bar{B} \bar{C}+\bar{C} \bar{B}=0$. From this relationship, a straightforward regrouping of terms shows that

$$
e^{(\bar{B}+\epsilon \bar{C}) \omega t}=e^{\bar{B} \omega t}+\epsilon \bar{C} \sin \omega t+o(\epsilon) .
$$

In terms of this expression, we write the solution of (7.9) as

$$
\left(\begin{array}{l}
u_{1}(t) \\
u_{2}(t)
\end{array}\right)=\left(e^{\bar{B} \omega t}+\epsilon \bar{C} \sin \omega t\right)\left(\begin{array}{l}
u_{1}(0) \\
u_{2}(0)
\end{array}\right)+o(\epsilon),
$$

and a straightforward calculation shows that this is equivalent to (7.8).

Lemma 6.1 enables a proof of the following.

Theorem 7.1. Let $B$ and $C$ be as in Lemma 6.1, and let $Y_{\epsilon}(t)$ be the solution of

$$
\dot{Y}(t)=\epsilon \Psi(t) Y(t), \quad Y(0)=I,
$$

where

$$
\Psi(t)=\sin \omega t e^{\left(B \omega-\Omega_{0}\right) t}\left[C e^{B \omega t} \Omega_{0} e^{-B \omega t}+e^{B \omega t} \Omega_{0} e^{-B \omega t} C\right] e^{\left(-B \omega+\Omega_{0}\right) t} .
$$

Let

$$
\hat{X}_{\epsilon}(t)=e^{B \omega t} e^{\left(-B \omega+\Omega_{0}\right) t} Y_{\epsilon}(t) .
$$


Then the solution $X_{\epsilon}(t)$ of (7.7) satisfies

$$
\left\|X_{\epsilon}(t)-\hat{X}_{\epsilon}(t)\right\| \sim \mathcal{O}(\epsilon)
$$

and this approximation remains valid over the time interval $[0,1 / \epsilon)$.

Proof. Equation (7.8) explicitly represents the effect of the phase perturbation on the evolution equation (7.4). In terms of this, we may describe the effect on the state evolution as follows. Let $\Phi(t)$ be the solution of

$$
\dot{X}(t)=e^{B \omega t} \Omega_{0} e^{-B \omega t} X(t), X(0)=I .
$$

From (2.11) this is given explicitly by

$$
\Phi(t)=e^{B \omega t} e^{\left(-B \omega+\Omega_{0}\right) t} .
$$

Given $X_{\epsilon}(t)$ defined by (7.7), let $Z(t)=\Phi(t)^{-1} X_{\epsilon}(t)$. Then $Z$ satisfies

$$
\dot{Z}(t)=\epsilon \sin \omega t\left[\Phi(t)^{-1}\left(C \Omega_{0} e^{-B \omega t}+e^{B \omega t} \Omega_{0} C\right) \Phi(t)\right] Z(t)+o(\epsilon) .
$$

The terms between the square brackets can be rewritten to give

$$
\dot{Z}(t)=\epsilon \Psi(t) Z(t)+o(\epsilon), \quad Z(0)=I .
$$

From this it follows that $Y(t)$ as defined in the theorem statement is an approximation of $Z(t)$, and the approximation result claimed for $X_{\epsilon}$ follows as a consequence.

REMARK 7.1. The earlier sections of the paper have posed control communication complexity problems for nonlinear systems through which controller-agents cooperatively steer the system toward various goal states by inputting different choices of control loop inputs. The results presented in Sections 4 and 5 cover ideal cases in which two agents (Alice and Bob) always execute loop inputs that are exactly ninety degrees out of phase. Theorem 6.1 addresses the question of how robust this mode of Alice and Bob's communicating will be in the case that an actual physical action is required in order to input the loop (e.g. controlling a ball between two plates or modulating an rf-field, as in Section 2). Theorem 6.1 expresses the effect of phase input uncertainty modeled in terms of a small constant phase error $\epsilon$. Over a single cycle of the inputs $u_{1}$ and $u_{2}(0 \leq t \leq 2 \pi / \omega)$, we expect that the state-trajectory generated by the input loops with the error term included will not deviate much from the ideal state-trajectory that has no phase error. Over many cycles, however, even a small phase error can lead to large deviation from the ideal - a phenomenon that might be referred to as phase-induced decoherence: the loss of state information with the passage of time. A complete analysis of decoherence due to misregistration of the amplitudes and phases of Alice and Bob's input loops is beyond the scope of the present paper. A typical situation for an $S^{2}$-system is illustrated in Fig. 4, however, wherein the thick trajectory corresponding to a phase-error $\epsilon=0.04$ is plotted 
along with the corresponding thin trajectory corresponding to no phase error (i.e. $\epsilon=0)$. The way in which uncertainty limits what can be communicated through the cooperative application of control inputs will be studied elsewhere.
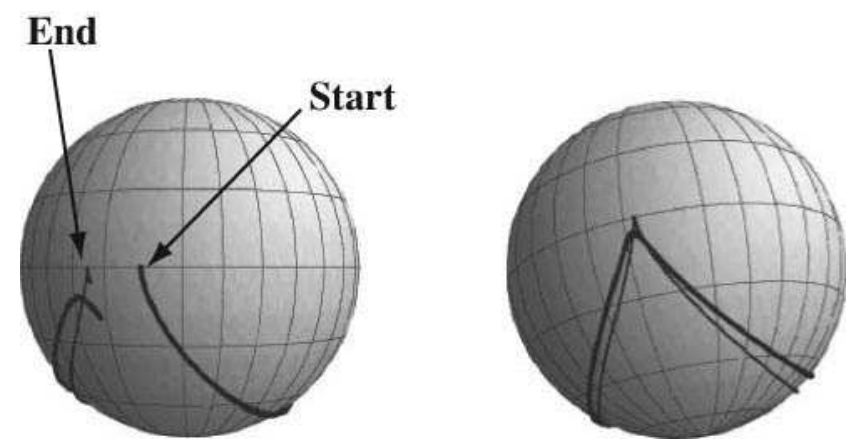

FIG. 4. Trajectories initiated at the point $(1,0,0)$ on the unit sphere $S^{2}$ and generated by the control inputs $u_{1}$ and $u_{2}$ of Lemma 6.1 applied to the system (2.13). Here $\omega=3, \lambda=\sqrt{4 \pi^{2}-\omega^{2}}$, $\varphi=0$, and $\epsilon=0$ for the thin black trajectory while $\epsilon=0.04$ for the thick black trajectory. The trajectories run for $0 \leq t \leq 2 \pi / \omega$ and are almost indistinguishable in the beginning and end at well separated locations on the sphere. (a) and (b) show the "front" and "rear" views.

8. Concluding Remarks. In this paper, the concept of control communication complexity is introduced for a family of nonlinear systems. These nonlinear systems arise in diverse contexts, such as classical mechanics, quantum computing, and nonholonomic systems of the type represented by the Brockett Integrator. The control communication complexity provides a means to measure the minimum information exchange required in order to achieve a specific control objective. The concept of control energy complexity and time complexity are also introduced. It is shown that there is an intricate relationship among all these complexity concepts as demonstrated by a few examples. This initial investigation points to potentially deeper results in several directions. One long-term objective is to discover and to understand fundamental connections among system dynamics, information coding, control function design, and optimization objectives. Connections with quantum control and quantum computing systems suggest another enticing course for further investigation.

\section{REFERENCES}

[Bal1] J. Baillieul, Geometric Methods for Optimal Control Problems, J. of Optimization Theory and Applications, 28:4(1978), pp. 519-548.

[Bal2] J. Baillieul And B. Lehman, Open-Loop Control Using Oscillatory Inputs, CRC Control Handbook, W.S. Levine, Editor, CRC Press, Boca Raton, FL, 1996, pp. 967-980.

[BaAn] J. Baillieul and P. Antsaklis, Control and Communication Challenges in Networked RealTime Systems, Proceedings of the IEEE, 95:1, January, 2007, pp. 9-28 Digital Object Identifier 10.1109/JPROC. 2006.887290. 
[BoEdBe] L. Bouten, S. Edwards \& V.P. Belavkin, Bellman equations for optimal feedback control of qubit states, in arXiv:quant-ph/0407192v2 21 Mar 2005.

[RWB0] R.W. Brockett, Lie Theory and Control Systems Defined on Spheres, SIAM J. Applied Math., 10:2(1973), pp. 213-225.

[RWB1] R.W. Brockett, Robotic Manipulators and the Product of Exponentials Formula, in: Mathematical Theory of Networks and Systems, Proceedings of the International Symposium Held at the Ben Gurion University of the Negev, Beer Sheva, June 20-24, 1983 (Ed. P. A. Fuhrmann). Berlin: Springer-Verlag, pp. 120-127, 1984.

[RWB2] R.W. Brockett, Asymptotic Stability and Feedback Stabilization, in: Differential Geometric Control Theory, R. W. Brockettt, R. S. Millman, and H. J. Sussman, Eds. Boston, Birkhauser, pp. 181-191, 1983.

[RWB3] R.W. Brockett, On the Rectification of Vibrating Motion, Sensors and Actuators, 20(1989), pp. 91-96.

[RWB4] R.W. Brockett, Minimizing attention in a motion control context, in: Proceedings of the 42nd IEEE Conference on Decision and Control, Dec. 9-12, 2003, Page(s):3349 - 3352 vol.4 Digital Object Identifier 10.1109/CDC.2003.1271661.

[RWB5] R.W. Brockett, Optimal Control of the Liouville Equation, Proceedings of the International Conference on Complex Geometry and Related Fields, (Zhijie Chen et al. Eds. ) American Mathematical Society, 2007, pp. 23-35.

[CaMa] M. Carmeli and S. Malin, Theory of Spinors: An Introduction, World Scientific Publishing, Singapore, ISBN 981-02-4261-1, 2000.

[Dal] D. D'Alessandro, Constructive Controllability of One and Two Spin $\frac{1}{2}$ Particles, Proceedings of the American Control Conference, Arlington, VA, June 25-27, 2001, pp. 1715-1720.

[DAiDa] D. D'Alessandro and M. Dahleh, Optimal Control of Two-Level Quantum Systems, IEEE Trans. on Automatic Control, 46:6(2001), pp. 866-876.

[Feynman] R.P. Feynman, Feynman Lectures on Computation, edited by Anthony J. G. Hey and Robin W. Allen, 1996, Addison-Wesley, ISBN 0-201-48991-0.

[Holevo] A.S. Holevo, Statistical Structure of Quantum Theory, Springer, New York, Springer-Verlag, Berlin, ISBN 3-540-42082-7, 2001.

[KuNi] E. Kushilevitz and N. Nisan, Communication Complexity, Cambridge University Press, Cambridge, UK, ISBN 0-521-56067-5 1997.

[LiKh] J.S. Li and N. Khaneja, Ensemble Controllability of the Bloch Equations, in: Proc. of the 45-th IEEE Conference on Decision and Control, San Diego, pp. 2483-2487, December 13-15, 2006.

[LiBa] K. Li AND J. BAIllieul, Robust quantization for digital finite communication bandwidth (DFCB) control, IEEE Trans. on Automatic Control, 49:9(2004), pp. 1573-1584.

[MaKha] H. Mabuchi And N. Khaneja, Principles and Applications of Control in Quantum Systems, Int'l J. of Robust and Nonlinear Control, 15(2005), pp. 647-667.

[Osserman] R. Osserman, The Isoperimetric Inequality, Bull. of American Math. Soc., 84:6(1978), pp. $1182-1238$.

[TaKr] C.J. Taylor and D.J. Kriegman, Minimization on the lie group so(3) and related manifolds. Technical Report 9405, Center for Systems Science, Dept. of Electrical Engineering, Yale University, New Haven, CT, April 1994.

[Tof] T. Toffoli, Bicontinuous Extensions of Invertible Combinatorial Functions, Mathematical Systems Theory, 14(1981), pp. 13-23.

[Var] V.S. Varadarajan, Lie Groups, Lie Algebras, and Their Representations, SpringerVerlag, New York, ISBN0-387-90969-9, 1984.

[Wong] W.S. Wong, Control Communication Complexity of Distributed Control Systems, to appear in SIAM Journal of Control and Optimization.

[WongBa1] W.S. Wong And J. Baillieul, Cost and Complexity of Distributed Choices, CUHK 
Preprint, July, 2008.

[WoBr1] W.S. Wong And R.W. Brockett, Systems with finite communication bandwidth constraints I: state estimation problems, IEEE Trans. on Automatic Control, 42:9(1997), pp. 1294-1298.

[WoBr2] W.S. Wong And R.W. Brockett, Systems with finite communication bandwidth constraints II: stabilization with limited information feedback, IEEE Trans. on Automatic Control, 44:5(1999), pp. 1049-1053.

[Yao1] A.C.-C. YAO, Some complexity questions related to distributive computing, in: Proceedings of the 11-th Annual ACM Symposium on Theory of Computing (STOC), 1979.

[Yao2] A.C.-C. YAO, Quantum Circuit Complexity, in: Proc. of the 34-th Annual IEEE Symposium on Foundations of Computer Science, pp. 352-361, November, 1993. 